\title{
THE TWO GRID STAGGERED SOLUTION ALGORITHM EXPLAINED
}

\section{Saumik Dana ${ }^{1}$}

${ }^{1}$ University of Southern California, Los Angeles, CA 90089

This document is a rendition of the two grid staggered solution algorithm for coupled flow and geomechanics

KEY WORDS: Two grid, finite element, coupled flow and geomechanics

\section{INTRODUCTION}

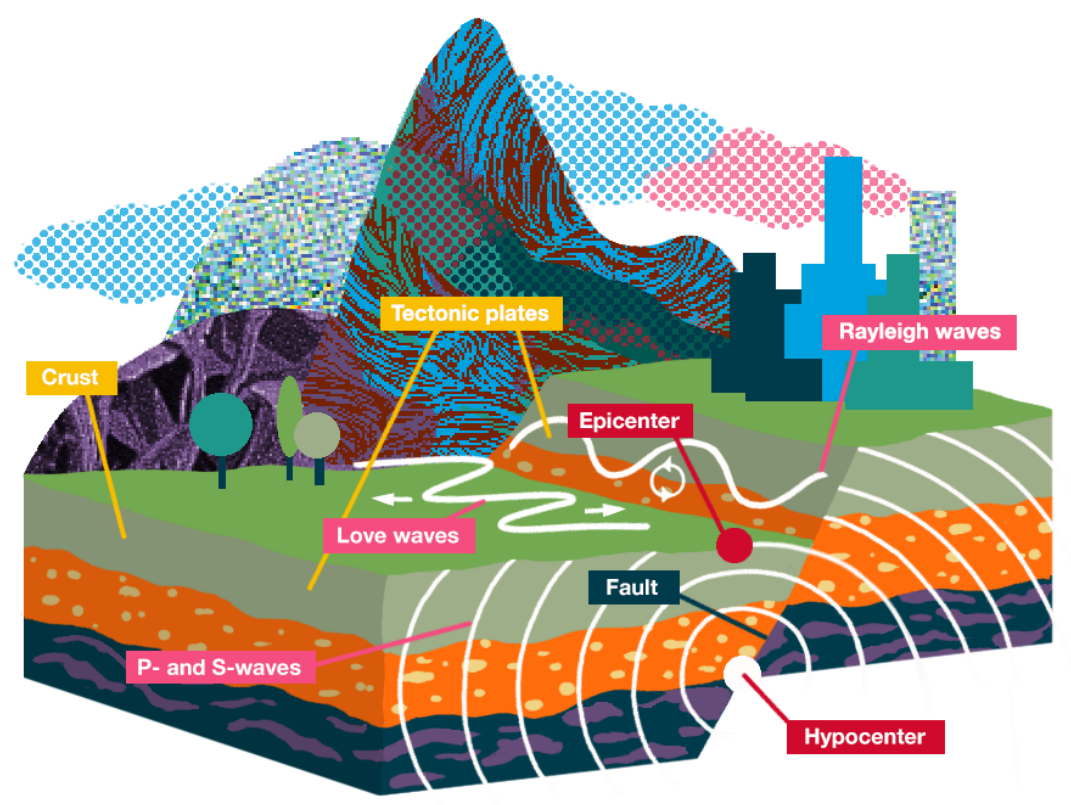

FIG. 1: Source: Caltech Science Exchange

The earthquake cycle, from slow deformation associated with interseismic behavior to rapid deformation associated with earthquake rupture, spans spatial scales ranging from fractions of a meter associated with the size of contact asperities on faults and individual grains to hundreds of kilometers associated with plate boundaries [1]. Similarly, temporal scales range from fractions of a second associated with slip at a point during earthquake rupture to hundreds of years of strain accumulation between earthquakes. In many cases, earthquakes are triggered after pore pressure perturbations activate critically stressed seismogenic faults [1], not just due to natural causes like earth tides [2], rainfall [3], snowfall [4], typhoons [5], but also due to human activity [6]. As faults slip, the induced stress field spawns 
seismic waves, which travel through and around the earth (see Fig. 1 and are recorded as ground displacement, velocity and/or acceleration time-series data at seismic stations. Understanding the causality between the events leading to fault slip and the recorded seismic data is important for seismic design and monitoring of underground structures [7] and reinforced concrete buildings [8] as well as climate mitigation projects like carbon sequestration [9] and energy technologies like enhanced geothermal systems [10] or oilfield wastewater disposal [11]. Earthquakes lead to losses to the tune of billions of dollars annually in the United States, with the FEMA and USGS pegging the number at \$6.1 billion for the year 2016. The number only includes direct economic losses to buildings, and does not cover damage and losses to critical facilities, transportation and utility lifelines or indirect economic losses. In quasi-static simulations [12-24], the effort is to couple the effects of pore pressure and quasi-static geomechanics, and while that effort is robust when the coupling is restricted to the reservoir, it is not robust when the coupling is scaled to the earth's surface. In this document, we provide a detailed rendition of the two-grid algorithm of [19], which was designed to tackle the scaling issue.

\section{MODEL EQUATIONS}

The equations for slightly single compressible single phase flow coupled with linear poromechanics are given in this module

\subsection{Flow model}

Let $\Omega^{f} \subset \mathbb{R}^{3}$ be the flow domain with boundary $\partial \Omega^{f}=\Gamma_{D}^{f} \cup \Gamma_{N}^{f}$ where $\Gamma_{D}^{f}$ is Dirichlet boundary and $\Gamma_{N}^{f}$ is Neumann boundary. The mass conservation equation (1) for single phase flow in porous medium* with the Darcy law (2) for slightly compressible fluid (3) with boundary conditions (4) and initial conditions (5) is

$$
\begin{aligned}
& \frac{\partial\left(\phi^{*} \rho\right)}{\partial t}+\nabla \cdot \mathbf{z}=q \\
& \mathbf{z}=-\frac{\mathbf{K} \rho}{\mu}(\nabla p-\rho \mathbf{g}) \\
& \rho=\rho_{0} e^{c\left(p-p_{0}\right)} \\
& p=g \text { on } \Gamma_{D}^{f} \times(0, T], \mathbf{z} \cdot \mathbf{n}=0 \text { on } \Gamma_{N}^{f} \times(0, T] \\
& p(\mathbf{x}, 0)=p_{0}(\mathbf{x}), \rho(\mathbf{x}, 0)=\rho_{0}(\mathbf{x}), \phi(\mathbf{x}, 0)=\phi_{0}(\mathbf{x}) \quad \forall \mathbf{x} \in \Omega^{f}
\end{aligned}
$$

where $p: \Omega^{f} \times(0, T] \rightarrow \mathbb{R}$ is the fluid pressure, $\mathbf{z}: \Omega^{f} \times(0, T] \rightarrow \mathbb{R}^{3}$ is the fluid flux, $\bar{\epsilon}$ is the volumetric strain, $\phi$ is the Eulerian porosity defined as the ratio of the pore volume in the deformed configuration to the total volume in the deformed configuration, $\phi^{*} \equiv \phi(1+\bar{\epsilon})$ is the Lagrangian porosity ${ }^{\dagger}$ defined as the ratio of the pore volume in the deformed configuration to the total volume in the undeformed configuration, $\mathbf{n}$ is the unit outward normal on $\Gamma_{N}^{f}, q$ is the source or sink term, $\mathbf{K}$ is the uniformly symmetric positive definite absolute permeability tensor, $\mu$ is the fluid viscosity, $\rho_{0}$ is a reference density, $c$ is the fluid compressibility and $T>0$ is the time interval.

\subsection{Poromechanics model}

Let $\Omega^{p} \subset \mathbb{R}^{3}$ be the poromechanics domain with boundary $\partial \Omega^{p}=\Gamma_{D}^{p} \cup \Gamma_{N}^{p}$ where $\Gamma_{D}^{p}$ is Dirichlet boundary and $\Gamma_{N}^{p}$ is Neumann boundary. The linear momentum balance in the quasi-static limit of interest (6) with the definition of the total stress (7) (see [26]) with the expression for the body force (8) and the small strain assumption (9) with boundary

\footnotetext{
* The derivation of the equation is given in [25]

${ }^{\dagger}$ The reader is again refered to [25] for a more detailed explanation of Eulerian and Lagrangian porosities
} 
conditions (10) and initial condition (11) is

$$
\begin{aligned}
& \nabla \cdot \boldsymbol{\sigma}+\mathbf{f}=0 \\
& \boldsymbol{\sigma}=\mathbb{D} \boldsymbol{\epsilon}-\alpha p \mathbf{I} \equiv \lambda \bar{\epsilon} \mathbf{I}+2 G \mathbf{\epsilon}-\alpha p \mathbf{I} \\
& \mathbf{f}=\rho \phi \mathbf{g}+\rho_{r}(1-\phi) \mathbf{g} \\
& \mathbf{\epsilon}(\mathbf{u})=\frac{1}{2}\left(\nabla \mathbf{u}+\nabla^{T} \mathbf{u}\right) \\
& \mathbf{u} \cdot \mathbf{n}_{1}=0 \text { on } \Gamma_{D}^{p} \times[0, T], \boldsymbol{\sigma}^{T} \mathbf{n}_{2}=\mathbf{t} \text { on } \Gamma_{N}^{p} \times[0, T] \\
& \mathbf{u}(\mathbf{x}, 0)=0 \quad \forall \mathbf{x} \in \Omega^{p}
\end{aligned}
$$

where $\mathbf{u}: \Omega^{p} \times[0, T] \rightarrow \mathbb{R}^{3}$ is the solid displacement, $\rho_{r}$ is the rock density, $G$ is the shear modulus, $\lambda$ is the Lame parameter, $\mathbf{n}_{1}$ is the unit outward normal to $\Gamma_{D}^{p}, \mathbf{n}_{2}$ is the unit outward normal to $\Gamma_{N}^{p}, \alpha$ is the Biot parameter,f is body force per unit volume, $t$ is the traction boundary condition, $\epsilon$ is the strain tensor, $\mathbb{D}$ is the fourth order elasticity tensor and $\mathbf{I}$ is the second order identity tensor.

\subsection{Expression for mean stress}

The mean stress $\sigma_{v}$ is given as

$$
\begin{aligned}
\sigma_{v} & =\frac{1}{3} \operatorname{tr}(\boldsymbol{\sigma})=\frac{1}{3} \operatorname{tr}(\lambda \bar{\epsilon} \mathbf{I}+2 G \boldsymbol{\epsilon}-\alpha p \mathbf{I})=\frac{1}{3} 3 \lambda \bar{\epsilon}+\frac{1}{3} 2 G \overbrace{\operatorname{tr}(\mathbf{\epsilon})}^{\bar{\epsilon}}-\frac{1}{3} 3 \alpha p \\
& =\left(\lambda+\frac{1}{3} 2 G\right) \bar{\epsilon}-\alpha p=K_{b} \bar{\epsilon}-\alpha p
\end{aligned}
$$

where $K_{b} \equiv \lambda+\frac{1}{3} 2 G$ is the bulk modulus of the solid skeleton or the drained bulk modulus.

\section{THE FIXED STRESS SPLIT STAGGERED SOLUTION STRATEGY}

Staggered solution schemes are those in which an operator splitting strategy is used to split the coupled problem into well-posed flow and mechanics subproblems which are then solved sequentially instead of solving the coupled system of equations monolithically. The fixed stress split is one such strategy that solves the flow problem while freezing the mean stress followed by the mechanics problem. This procedure (also refered to as 'coupling iteration' or 'fixed - stress iteration') is repeated until a certain convergence criterion is met. The basic idea of the fixed stress split strategy is to solve for the pressure and flux degrees of freedom at the current fixed - stress iteration based on the value of the mean stress from the previous fixed - stress iteration. These pressures then contribute to the force vector in the poromechanics system which is solved for displacements thereby updating the stress state. This updated stress state is then fed back to the flow system for the next fixed - stress iteration. Since this strategy condemns the porous solid to follow a certain stress path during the flow solve, the convergence of the solution algorithm is not automatically guaranteed. [27] proved the convergence of the fixed - stress split strategy using the principle of contraction mapping with appropriately chosen metrics. [28] showed that the fixed stress split strategy can be interpreted as a block triangular preconditioning strategy applied within a Richardson iteration to the fully implicit scheme. They also showed that a mixed formulation for flow with the fixed mean stress constraint coupled with a continuous Galerkin formulation for poromechanics is first order accurate in space and time for both pressure and displacement, even for distorted meshes.

\section{DISCRETE VARIATIONAL STATEMENTS}

Given a domain $\Omega \subset \mathbb{R}^{n}$, we use $\mathbb{P}_{k}(\Omega)$ to represent the restriction of the space of polynomials of degree less that or equal to $k$ to $\Omega$. Given a domain $\Omega \subset \mathbb{R}^{3}$, we use $\mathbb{Q}_{1}(\Omega)$ to denote the space of trilinears ${ }^{\ddagger}$ on $\Omega$. The Sobolev spaces

\footnotetext{
${ }^{\ddagger}$ If $\Omega \subset \mathbb{R}^{2}, \mathbb{Q}_{1}(\Omega)$ is used to denote the space of bilinears on $\Omega$.
} 
([29]) are based on the space of square integrable functions on a domain $\Omega \subset \mathbb{R}^{3}$ given by

$$
L^{2}(\Omega) \equiv\left\{f: \int_{\Omega}|f|^{2}=\|f\|_{L^{2}(\Omega)}^{2}<+\infty\right\},
$$

We then define in general, for any integer $m \geq 0$

$$
H^{m}(\Omega) \equiv\left\{w: D^{\alpha} w \in L^{2}(\Omega) \forall|\alpha| \leq m\right\}
$$

where the derivatives are taken in the sense of distributions and given by

$$
D^{\alpha} w=\frac{\partial^{|\alpha|} w}{\partial x_{1}^{\alpha_{1}} . . \partial x_{n}^{\alpha_{n}}},|\alpha|=\alpha_{1}+\cdots+\alpha_{n},
$$

In the presence of a divergence operator in second order PDEs, the following space is defined

$$
\mathbf{H}(\operatorname{div}, \Omega) \equiv\left\{\mathbf{v}: \mathbf{v} \in\left(L^{2}(\Omega)\right)^{3}, \nabla \cdot \mathbf{v} \in L^{2}(\Omega)\right\}
$$

For $\Omega \subset \mathbb{R}^{3}$ and (.) defined on $\Omega$, we omit the differential $d V$ in the representation of the integral of $(\cdot)$ over $\Omega$ for the sake of brevity as follows

$$
\int_{\Omega}(\cdot) \equiv \int_{\Omega}(\cdot) d V
$$

For $\Omega \subset \mathbb{R}^{2}$ and $(\cdot)$ defined on $\Omega$, we omit the differential $d S$ in the representation of the integral of $(\cdot)$ over $\Omega$ for the sake of brevity as follows

$$
\int_{\Omega}(\cdot) \equiv \int_{\Omega}(\cdot) d S
$$

\subsection{Finite element mapping}

Let $T_{h}^{f}$ be the finite element partition of $\Omega^{f}$ consisting of distorted hexahedral elements $E^{f}$ and let $T_{H}^{p}$ be the finite element partition of $\Omega^{p}$ consisting of distorted hexahedral elements $E^{p}$. As the flow model is solved on a finer mesh, the mesh ratio $r$ is such that

$$
r \equiv \max _{E^{p} \in T_{H}^{p}} \operatorname{diam}\left(E^{p}\right) / \max _{E^{f} \in T_{h}^{f}} \operatorname{diam}\left(E^{f}\right)=H / h \geq 1
$$

Let $\mathbf{r}_{i}^{f}, i=1, . ., 8$ and $\mathbf{r}_{i}^{p}, i=1, . ., 8$ be the vertices of $E^{f}$ and $E^{p}$ respectively. Let $\hat{E}$ represent a reference unit cube with vertices $\hat{\mathbf{r}}_{1} \equiv(0,0,0), \hat{\mathbf{r}}_{2} \equiv(1,0,0), \hat{\mathbf{r}}_{3} \equiv(1,1,0), \hat{\mathbf{r}}_{4} \equiv(0,1,0), \hat{\mathbf{r}}_{5} \equiv(0,0,1), \hat{\mathbf{r}}_{6} \equiv(1,0,1), \hat{\mathbf{r}}_{7} \equiv(1,1,1)$ and $\hat{\mathbf{r}}_{8} \equiv(0,1,1)$. Letting $\hat{\mathbf{x}}=(\hat{x}, \hat{y}, \hat{z}) \in \hat{E}$, the functions $F_{E}^{f}(\hat{\mathbf{x}}): \hat{E} \mapsto E^{f}$ and $F_{E}^{p}(\hat{\mathbf{x}}): \hat{E} \mapsto E^{p}$ as shown in Figure 2 are defined as

$$
\begin{aligned}
F_{E}^{f}(\hat{\mathbf{x}}) & =\mathbf{r}_{1}^{f}(1-\hat{x})(1-\hat{y})(1-\hat{z})+\mathbf{r}_{2}^{f} \hat{x}(1-\hat{y})(1-\hat{z})+\mathbf{r}_{3}^{f} \hat{x} \hat{y}(1-\hat{z}) \\
& +\mathbf{r}_{4}^{f}(1-\hat{x}) \hat{y}(1-\hat{z})+\mathbf{r}_{5}^{f}(1-\hat{x})(1-\hat{y}) \hat{z}+\mathbf{r}_{6}^{f} \hat{x}(1-\hat{y}) \hat{z} \\
& +\mathbf{r}_{7}^{f} \hat{x} \hat{y} \hat{z}+\mathbf{r}_{8}^{f}(1-\hat{x}) \hat{y} \hat{z} \\
F_{E}^{p}(\hat{\mathbf{x}}) & =\mathbf{r}_{1}^{p}(1-\hat{x})(1-\hat{y})(1-\hat{z})+\mathbf{r}_{2}^{p} \hat{x}(1-\hat{y})(1-\hat{z})+\mathbf{r}_{3}^{p} \hat{x} \hat{y}(1-\hat{z}) \\
& +\mathbf{r}_{4}^{p}(1-\hat{x}) \hat{y}(1-\hat{z})+\mathbf{r}_{5}^{p}(1-\hat{x})(1-\hat{y}) \hat{z}+\mathbf{r}_{6}^{p} \hat{x}(1-\hat{y}) \hat{z} \\
& +\mathbf{r}_{7}^{p} \hat{x} \hat{y} \hat{z}+\mathbf{r}_{8}^{p}(1-\hat{x}) \hat{y} \hat{z}
\end{aligned}
$$



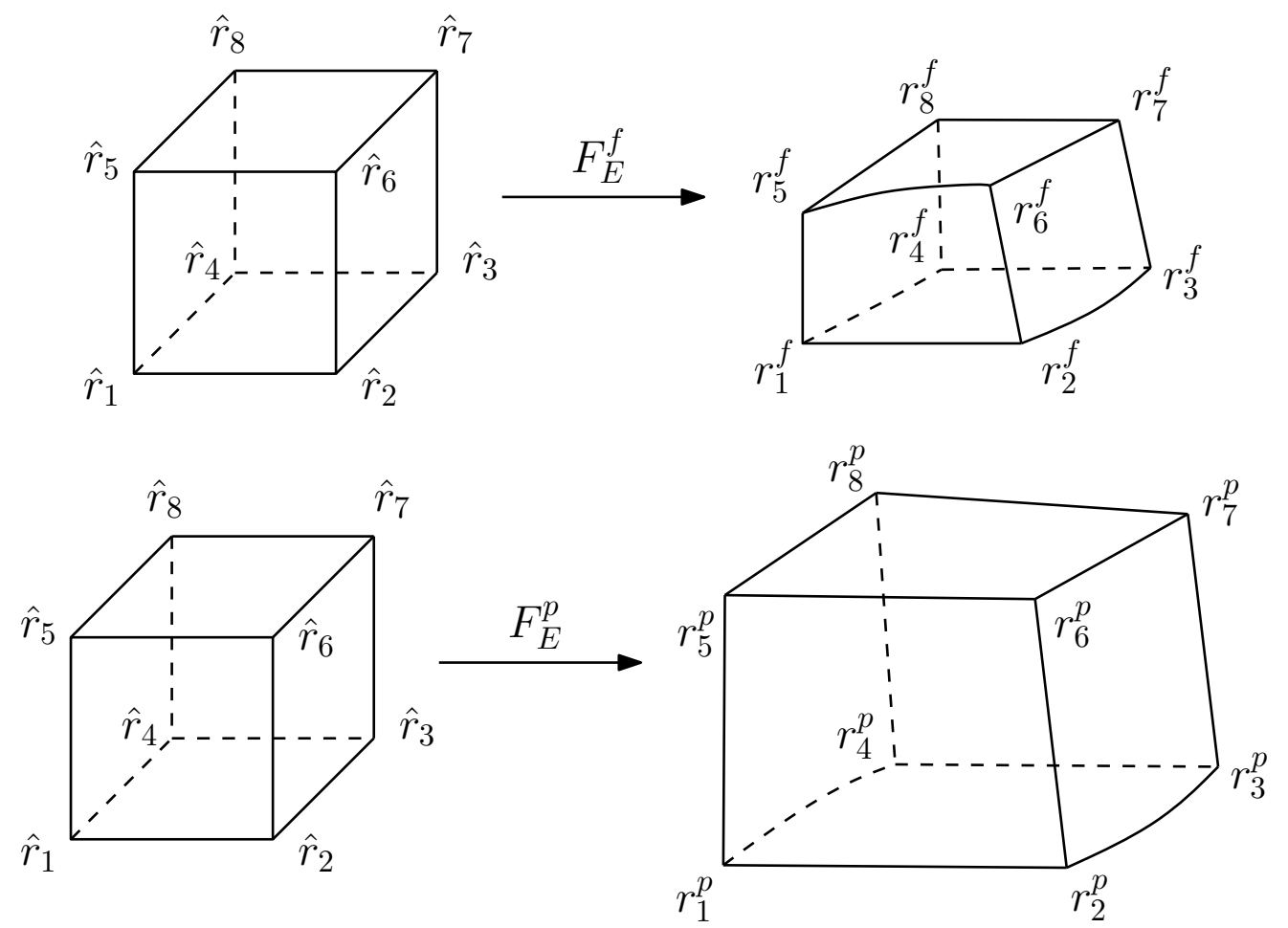

FIG. 2: Trilinear mapping $F_{E}^{f}: \hat{E} \mapsto E^{f}$ and $F_{E}^{p}: \hat{E} \mapsto E^{p}$ for 8 noded distorted hexahedral elements $E^{f}$ and $E^{p}$ respectively. The faces of $E^{f}$ and $E^{p}$ can be non-planar.

We denote the jacobian of $F_{E}^{f}$ and $F_{E}^{p}$ by $D F_{E}^{f}$ and $D F_{E}^{p}$ respectively and the determinant of $D F_{E}^{f}$ and $D F_{E}^{p}$ by $J_{E}^{f}$ and $J_{E}^{p}$ respectively. Defining $\mathbf{r}_{i j}^{f} \equiv \mathbf{r}_{i}^{f}-\mathbf{r}_{j}^{f}$ and $\mathbf{r}_{i j}^{p} \equiv \mathbf{r}_{i}^{p}-\mathbf{r}_{j}^{p}$, we have

$$
\begin{aligned}
D F_{E}^{f}(\hat{\mathbf{x}})= & {\left[\begin{array}{l}
\mathbf{r}_{21}^{f}+\left(\mathbf{r}_{34}^{f}-\mathbf{r}_{21}^{f}\right) \hat{y}+\left(\mathbf{r}_{65}^{f}-\mathbf{r}_{21}^{f}\right) \hat{z}+\left(\left(\mathbf{r}_{21}^{f}-\mathbf{r}_{34}^{f}\right)-\left(\mathbf{r}_{65}^{f}-\mathbf{r}_{78}^{f}\right)\right) \hat{y} ; \\
\mathbf{r}_{41}^{f}+\left(\mathbf{r}_{34}^{f}-\mathbf{r}_{21}^{f}\right) \hat{x}+\left(\mathbf{r}_{85}^{f}-\mathbf{r}_{41}^{f}\right) \hat{z}+\left(\left(\mathbf{r}_{21}^{f}-\mathbf{r}_{34}^{f}\right)-\left(\mathbf{r}_{65}^{f}-\mathbf{r}_{78}^{f}\right)\right) \hat{x} \hat{z} ; \\
\mathbf{r}_{51}^{f}+\left(\mathbf{r}_{65}^{f}-\mathbf{r}_{21}^{f}\right) \hat{x}+\left(\mathbf{r}_{85}^{f}-\mathbf{r}_{41}^{f}\right) \hat{y}+\left(\left(\mathbf{r}_{21}^{f}-\mathbf{r}_{34}^{f}\right)-\left(\mathbf{r}_{65}^{f}-\mathbf{r}_{78}^{f}\right)\right) \hat{x} \hat{y}
\end{array}\right]_{3 \times 3} } \\
D F_{E}^{p}(\hat{\mathbf{x}})= & {\left[\begin{array}{c}
\mathbf{r}_{21}^{p}+\left(\mathbf{r}_{34}^{p}-\mathbf{r}_{21}^{p}\right) \hat{y}+\left(\mathbf{r}_{65}^{p}-\mathbf{r}_{21}^{p}\right) \hat{z}+\left(\left(\mathbf{r}_{21}^{p}-\mathbf{r}_{34}^{p}\right)-\left(\mathbf{r}_{65}^{p}-\mathbf{r}_{78}^{p}\right) \hat{y} \hat{z} ;\right. \\
\mathbf{r}_{41}^{p}+\left(\mathbf{r}_{34}^{p}-\mathbf{r}_{21}^{p} \hat{x}+\left(\mathbf{r}_{85}^{p}-\mathbf{r}_{41}^{p}\right) \hat{z}+\left(\left(\mathbf{r}_{21}^{p}-\mathbf{r}_{34}^{p}\right)-\left(\mathbf{r}_{65}^{p}-\mathbf{r}_{78}^{p}\right) \hat{x} \hat{z} ;\right.\right. \\
\mathbf{r}_{51}^{p}+\left(\mathbf{r}_{65}^{p}-\mathbf{r}_{21}^{p}\right) \hat{x}+\left(\mathbf{r}_{85}^{p}-\mathbf{r}_{41}^{p}\right) \hat{y}+\left(\left(\mathbf{r}_{21}^{p}-\mathbf{r}_{34}^{p}\right)-\left(\mathbf{r}_{65}^{p}-\mathbf{r}_{78}^{p}\right)\right) \hat{x} \hat{y}
\end{array}\right]_{3 \times 3} }
\end{aligned}
$$

Let $\mathbf{x}^{f} \in E^{f}$ and $\mathbf{x}^{p} \in E^{p}$ and further let $\psi\left(\mathbf{x}^{f}\right)$ and $\psi\left(\mathbf{x}^{p}\right)$ be functions defined on $E^{f}$ and $E^{p}$ respectively. Let $\hat{\psi}(\hat{\mathbf{x}})$ be the corresponding definitions on $\hat{E}$. Then we have

$$
\begin{aligned}
& \nabla \psi\left(\mathbf{x}^{f}\right)=\left(D F_{E}^{f}\right)^{-T} \hat{\nabla} \hat{\psi} \\
& \nabla \psi\left(\mathbf{x}^{p}\right)=\left(D F_{E}^{p}\right)^{-T} \hat{\nabla} \hat{\psi}
\end{aligned}
$$




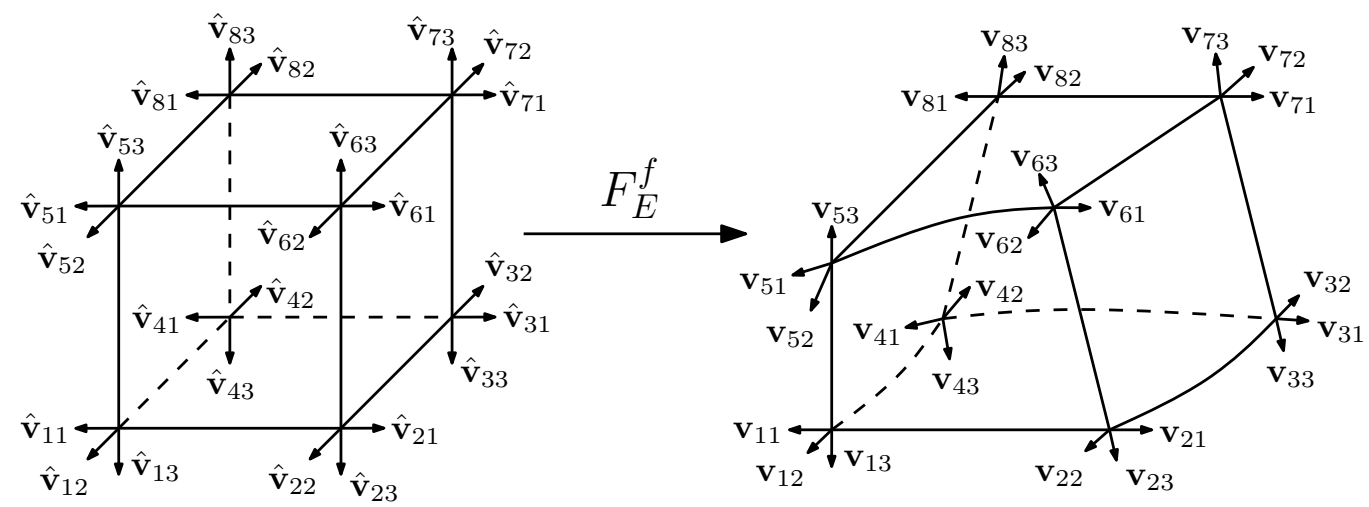

FIG. 3: Degrees of freedom and basis functions for the enhanced $\mathrm{BDDF}_{1}$ velocity space on hexahedra.

\subsection{Mixed finite element spaces employed}

Let $\mathbf{V}_{h}^{*} \times W_{h}$ be the lowest order $\mathrm{BDDF}_{1}$ MFE spaces on hexahedra (see [30]). With $\mathbf{x} \equiv(\hat{x}, \hat{y}, \hat{z}) \in \hat{E}$, these spaces are defined on $\hat{E}$ as

$$
\begin{aligned}
\hat{\mathbf{V}}^{*}(\hat{E}) & =\left(\mathbb{P}_{1}(\hat{E})\right)^{3}+r_{0} \operatorname{curl}(0,0, \hat{x} \hat{y} \hat{z})^{T}+r_{1} \operatorname{curl}\left(0,0, \hat{x} \hat{y}^{2}\right)^{T}+s_{0} \operatorname{curl}(\hat{x} \hat{y} \hat{z}, 0,0)^{T} \\
& +s_{1} \operatorname{curl}\left(\hat{y} \hat{z}^{2}, 0,0\right)^{T}+t_{0} \operatorname{curl}(0, \hat{x} \hat{y} \hat{z}, 0)^{T}+t_{1} \operatorname{curl}\left(0, \hat{x}^{2} \hat{z}, 0\right)^{T} \\
\hat{W}(\hat{E}) & =\mathbb{P}_{0}(\hat{E})
\end{aligned}
$$

with the following properties

$$
\hat{\nabla} \cdot \hat{\mathbf{V}}^{*}(\hat{E})=\hat{W}(\hat{E}), \quad \text { and } \quad \forall \hat{\mathbf{v}} \in \hat{\mathbf{V}}^{*}(\hat{E}), \forall \hat{e} \subset \partial \hat{E}, \hat{\mathbf{v}} \cdot \hat{\mathbf{n}}_{\hat{e}} \in \mathbb{P}_{1}(\hat{e})
$$

where $\hat{e}$ represents a face of $\hat{E}$ and $\hat{\mathbf{n}}_{\hat{e}}$ the unit outward normal to $\hat{e}$. The multipoint flux approximation procedure requires on each face one velocity degree of freedom to be associated with each vertex thus requiring four degrees of freedom per face. Since $\mathbf{V}_{h}^{*}$ has only three degrees of freedom per face, it is augmented with one degree of freedom per face resulting in addition of six degrees of freedom per element. Since the properties of constant divergence, linear independence of the shape functions and continuity of the normal component across the element faces are to be preserved, six curl terms are added (see [31]) to $\mathbf{V}_{h}^{*}$. Let $\mathbf{V}_{h} \times W_{h}$ be the enhanced BDDF 1 spaces on hexahedra. On $\hat{E}$, these spaces are

$$
\begin{aligned}
\hat{\mathbf{V}}(\hat{E}) & =\hat{\mathbf{V}}^{*}(\hat{E})+r_{2} \operatorname{curl}\left(0,0, \hat{x}^{2} \hat{z}\right)^{T}+r_{3} \operatorname{curl}\left(0,0, \hat{x}^{2} \hat{y} \hat{z}\right)^{T}+s_{2} \operatorname{curl}\left(\hat{x} \hat{y}^{2}, 0,0\right)^{T} \\
& +s_{3} \operatorname{curl}\left(\hat{x} \hat{y}^{2} \hat{z}^{2}, 0,0\right)^{T}+t_{2} \operatorname{curl}\left(0, \hat{y} \hat{z}^{2}, 0\right)^{T}+t_{3} \operatorname{curl}\left(0, \hat{x} \hat{y} \hat{z}^{2}, 0\right)^{T} \\
\hat{W}(\hat{E}) & =\mathbb{P}_{0}(\hat{E})
\end{aligned}
$$

with the following properties

$$
\hat{\nabla} \cdot \hat{\mathbf{V}}(\hat{E})=\hat{W}(\hat{E}), \quad \text { and } \quad \forall \hat{\mathbf{v}} \in \hat{\mathbf{V}}(\hat{E}), \forall \hat{e} \subset \partial \hat{E}, \hat{\mathbf{v}} \cdot \hat{\mathbf{n}}_{\hat{e}} \in \mathbb{Q}_{1}(\hat{e})
$$

Since $\operatorname{dim} \mathbb{Q}_{1}(\hat{e})=4$, the dimension of $\hat{\mathbf{V}}(\hat{E})$ is 24 as shown in Figure 3 .

\subsection{Weak form of Darcy law}

We first rewrite the Darcy law (2) as

$$
\frac{\mu}{\rho} \mathbf{K}^{-1} \mathbf{z}=-\nabla p+\rho \mathbf{g}
$$


The weak form of the above is

$$
\int_{\Omega^{f}} \frac{\mu}{\rho} \mathbf{K}^{-1} \mathbf{z} \cdot \mathbf{v}=-\int_{\Omega^{f}} \nabla p \cdot \mathbf{v}+\int_{\Omega^{f}} \rho \mathbf{g} \cdot \mathbf{v}
$$

We use the divergence theorem for the first term on RHS of (17) as follows

$$
\begin{aligned}
& \int_{\Omega^{f}} \nabla p \cdot \mathbf{v}=\int_{\Omega^{f}}(\nabla \cdot(p \mathbf{v})-p \nabla \cdot \mathbf{v}) \\
& =\int_{\partial \Omega^{f}} p \mathbf{v} \cdot \mathbf{n}-\int_{\Omega^{f}} p \nabla \cdot \mathbf{v} \\
& =\int_{\Gamma_{D}^{f}} g \mathbf{v} \cdot \mathbf{n}-\int_{\Omega^{f}} p \nabla \cdot \mathbf{v}
\end{aligned}
$$

where we invoke $\mathbf{v} \cdot \mathbf{n}=0$ on $\Gamma_{N}^{f}$. Substituting (18) in (17), we get

$$
\int_{\Omega^{f}} \frac{\mu}{\rho} \mathbf{K}^{-1} \mathbf{z} \cdot \mathbf{v}-\int_{\Omega^{f}} p \nabla \cdot \mathbf{v}=\int_{\Omega^{f}} \rho \mathbf{g} \cdot \mathbf{v}-\int_{\Gamma_{D}^{f}} g \mathbf{v} \cdot \mathbf{n}
$$

\subsection{Discrete weak form of flow model}

A subspace of $\mathbf{H}\left(\operatorname{div}, \Omega^{f}\right)$ consisting of functions with vanishing normal trace on $\Gamma_{N}^{f}$ is

$$
\mathbf{V}\left(\Omega^{f}\right) \equiv \mathbf{H}\left(\operatorname{div}, \Omega^{f}\right) \cap\left\{\mathbf{v}: \mathbf{v} \cdot \mathbf{n}=0 \text { on } \Gamma_{N}^{f}\right\}
$$

The problem statement is : find $\mathbf{z}_{h}, \tilde{\mathbf{z}}_{h} \in \mathbf{V}_{h}$ and $p_{h} \in W_{h}$ such that

$$
\begin{aligned}
& \sum_{E^{f} \in T_{h}^{f}} \int_{E^{f}} \frac{\mu}{\rho} \mathbf{K}^{-1} \mathbf{z}_{h} \cdot \mathbf{v}-\sum_{E^{f} \in T_{h}^{f} E^{f}} \int_{h} \nabla \cdot \mathbf{v} \\
& =-\sum_{E^{f} \in T_{h}^{f} \partial E^{f} \cap \Gamma_{D}^{f}} g \mathbf{v} \cdot \mathbf{n}+\sum_{E^{f} \in T_{h}^{f} E^{f}} \rho \mathbf{g} \cdot \mathbf{v} \\
& \sum_{E^{f} \in T_{h}^{f}} \int_{E^{f}} \phi^{*} \rho w+\sum_{E^{f} \in T_{h}^{f} E^{f}} \Delta t \nabla \cdot \mathbf{z}_{h} w \\
& =\sum_{E^{f} \in T_{h}^{f}} \int_{E^{f}} \Delta t q w+\sum_{E^{f} \in T_{h}^{f} E^{f}} \int\left(\phi^{*} \rho\right)^{n} w
\end{aligned}
$$

where the finite dimensional subspaces $W_{h} \subset L^{2}\left(\Omega^{f}\right)$ and $\mathbf{V}_{h} \subset \mathbf{V}\left(\Omega^{f}\right)$ are

$$
\begin{aligned}
& W_{h} \equiv\left\{w:\left.w\right|_{E^{f}} \in \mathbb{P}_{0}\left(E^{f}\right) \forall E^{f} \in T_{h}^{f}\right\} \\
& \mathbf{V}_{h} \equiv\left\{\mathbf{v}:\left.\left.\mathbf{v}\right|_{E^{f}} \leftrightarrow \hat{\mathbf{v}}\right|_{\hat{E}}:\left.\hat{\mathbf{v}}\right|_{\hat{E}} \in \hat{\mathbf{V}}(\hat{E}) \forall E^{f} \in T_{h}^{f}, \mathbf{v} \cdot \mathbf{n}=0 \text { on } \Gamma_{N}^{f}\right\}
\end{aligned}
$$


$(\cdot)^{n}$ denotes the quantity $(\cdot)$ evaluated at the previous time level $n$ while the remaining terms are evaluated at current time level $n+1$. Equations (20)-(21) are recast for the $(k+1)^{t h}$ Newton iteration as

$$
\left[\begin{array}{cc}
\mathbf{A} & -\mathbf{B} \\
\Delta t \mathbf{B}^{T} & \mathbf{C}
\end{array}\right]\left\{\begin{array}{l}
\delta^{(k)} \mathbf{Z}_{h} \\
\delta^{(k)} p_{h}
\end{array}\right\}=\left\{\begin{array}{l}
R_{1} \\
R_{2}
\end{array}\right\}
$$

where $\mathbf{Z}_{h}$ are the flux degrees of freedom and $p_{h}$ are the pressure degrees of freedom respectively and the submatrices $\mathbf{A}, \mathbf{B}$ and $\mathbf{C}$ are

$$
\begin{aligned}
& \mathbf{A}=\sum_{E^{f} \in T_{h}^{f}} \int_{E^{f}} \frac{\mu}{\rho^{k}} \mathbf{K}^{-1} \mathbf{v}_{j} \cdot \mathbf{v}_{i} \\
& \mathbf{B}=\sum_{E^{f} \in T_{h}^{f} E_{f}^{f}} \nabla \cdot \mathbf{v}_{i} w_{j} \\
& \mathbf{C}=\sum_{E^{f} \in T_{h}^{f}} \int_{E^{f}} \rho^{k} \phi^{*^{k}} c+\left.\sum_{E^{f} \in T_{h}^{f} E^{f}} \rho^{k} \frac{\partial \phi^{*}}{\partial p}\right|_{k}
\end{aligned}
$$

and the residuals $R_{1}$ and $R_{2}$ are

$$
\begin{aligned}
R_{1}= & -\sum_{E^{f} \in T_{h}^{f}} \int_{E^{f}} \mathbf{K}^{-1} \tilde{\mathbf{z}}_{h}^{k} \cdot \mathbf{v}+\sum_{E^{f} \in T_{h}^{f} E^{f}} \int_{h} p_{h}^{k} \nabla \cdot \mathbf{v} \\
& -\sum_{E^{f} \in T_{h}^{f}} \int_{E^{f} \cap \Gamma_{D}^{f}} g \mathbf{v} \cdot \mathbf{n}+\sum_{E^{f} \in T_{h}^{f} E^{f}} \int \rho^{k} \mathbf{g} \cdot \mathbf{v} \\
R_{2}= & -\sum_{E^{f} \in T_{h}^{f}} \int_{E^{f}} \phi^{*^{k}} \rho^{k} w-\sum_{E f \in T_{h}^{f} E^{f}} \int_{E^{f} \in T_{h}^{f}} \Delta t \nabla \cdot \mathbf{z}_{h}^{k} w \\
& +\sum_{E^{f} \in T_{h}^{f}} \Delta t q w+\sum_{E^{f}}\left(\phi^{*} \rho\right)^{n} w
\end{aligned}
$$

where $(\cdot)^{k}$ is the quantity $(\cdot)$ evaluated at the $k^{\text {th }}$ Newton iteration at the current time level $n+1$ and $\delta^{(k)}$ is the change in $(\cdot)$ over the $(k+1)^{t h}$ iteration. The submatrices $\mathbf{A}$ and $\mathbf{B}$ have off-diagonal components while the submatrix $\mathbf{C}$ is diagonal. The evaluation of $\left.\frac{\partial \phi^{*}}{\partial p}\right|_{k}$ in (23) along with the evolution law for $\phi^{*}$ during the flow solve are presented in the module 4.7. A quadrature rule ([31]) is used to evaluate the submatrix A. Elimination of the flux degrees of freedom from (22) results in a 27 point stencil where the pressure in each element $E^{f}$ is coupled with pressures in all 27 elements that share a vertex with $E^{f}$. The resulting system is solved for $\delta^{(k)} p_{h}$ and the $k^{\text {th }}$ iterate is obtained as

$$
p_{h}^{k+1}=p_{h}^{k}+\delta^{(k)} p_{h}
$$

\subsection{Discrete weak form of linear momentum balance}

An infinite dimensional space $\mathbf{U}\left(\Omega^{p}\right)$ in $\Omega^{p}$ is defined as

$$
\mathbf{U}\left(\Omega^{p}\right) \equiv\left\{\mathbf{q}=(u, v, w): u, v, w \in H^{1}\left(\Omega^{p}\right), \mathbf{q}=0 \text { on } \Gamma_{D}^{p}\right\}
$$

The weak form of the linear momentum balance (6) is given by

$$
\int_{\Omega^{p}}(\nabla \cdot \boldsymbol{\sigma}) \cdot \mathbf{q}+\int_{\Omega^{p}} \mathbf{f} \cdot \mathbf{q}=0 \quad\left(\forall \mathbf{q} \in \mathbf{U}\left(\Omega^{p}\right)\right)
$$


where $\mathbf{U}\left(\Omega^{p}\right)$ is given by

$$
\mathbf{U}\left(\Omega^{p}\right) \equiv\left\{\mathbf{q}=(u, v, w): u, v, w \in H^{1}\left(\Omega^{p}\right), \mathbf{q}=0 \text { on } \Gamma_{D}^{p}\right\}
$$

We know from tensor calculus that

$$
(\nabla \cdot \boldsymbol{\sigma}) \cdot \mathbf{q} \equiv \nabla \cdot(\boldsymbol{\sigma q})-\boldsymbol{\sigma}: \nabla \mathbf{q}
$$

Further, using the divergence theorem and the symmetry of $\sigma$, we arrive at

$$
\int_{\Omega^{p}} \nabla \cdot(\boldsymbol{\sigma q}) \equiv \int_{\partial \Omega^{p}} \mathbf{q} \cdot(\boldsymbol{\sigma n})
$$

We decompose $\nabla \mathbf{q}$ into a symmetric part $(\nabla \mathbf{q})_{s} \equiv \frac{1}{2}\left(\nabla \mathbf{q}+(\nabla \mathbf{q})^{T}\right) \equiv \mathbf{\epsilon}(\mathbf{q})$ and skew-symmetric part $(\nabla \mathbf{q})_{s s}$ and note that the contraction between a symmetric and skew-symmetric tensor is zero to obtain

$$
\boldsymbol{\sigma}: \nabla \mathbf{q} \equiv \boldsymbol{\sigma}:(\nabla \mathbf{q})_{s}+0 \boldsymbol{\sigma}:(\nabla \mathbf{q})_{s s}=\boldsymbol{\sigma}: \boldsymbol{\epsilon}(\mathbf{q})
$$

From (27), (28), (29) and (30), we get

$$
\int_{\partial \Omega^{p}}(\boldsymbol{\sigma n}) \cdot \mathbf{q}-\int_{\Omega^{p}} \boldsymbol{\sigma}: \mathbf{\epsilon}(\mathbf{q})+\int_{\Omega^{p}} \mathbf{f} \cdot \mathbf{q}=0
$$

which, after invoking the boundary conditions $\mathbf{\sigma n}=\mathbf{t}$ on $\Gamma_{N}^{p}$ and $\mathbf{q}=0$ on $\Gamma_{D}^{p}$ results in

$$
\int_{\Gamma_{N}^{p}} \mathbf{t} \cdot \mathbf{q}-\int_{\Omega^{p}} \boldsymbol{\sigma}: \mathbf{\epsilon}(\mathbf{q})+\int_{\Omega^{p}} \mathbf{f} \cdot \mathbf{q}=0
$$

Substituting $\boldsymbol{\sigma}=\mathbb{D} \boldsymbol{\epsilon}-\alpha p \mathbf{I}$ in (31), we get

$$
\int_{\Gamma_{N}^{p}} \mathbf{t} \cdot \mathbf{q}-\int_{\Omega^{p}}(\mathbb{D} \boldsymbol{\epsilon}-\alpha p \mathbf{I}): \boldsymbol{\epsilon}(\mathbf{q})+\int_{\Omega^{p}} \mathbf{f} \cdot \mathbf{q}=0
$$

and the discrete weak form after substituting $\mathbf{f}=\rho \phi \mathbf{g}+\rho_{r}(1-\phi) \mathbf{g}$ is finally obtained as: find $\mathbf{u}_{H} \in \mathbf{U}_{H}$ such that

$$
\begin{aligned}
& \sum_{E^{f} \in T_{H}^{p}} \int_{E^{p}} \boldsymbol{\epsilon}(\mathbf{q}): \mathbb{D} \boldsymbol{\epsilon}\left(\mathbf{u}_{H}\right)=\sum_{E^{f} \in T_{H}^{p} \Omega^{p}} \int_{\boldsymbol{c}} \boldsymbol{\epsilon}(\mathbf{q}): \alpha p \mathbf{I} \\
& +\sum_{E^{f} \in T_{H}^{p}} \int_{E^{p}} \mathbf{q} \cdot\left(\rho \phi \mathbf{g}+\rho_{r}(1-\phi) \mathbf{g}\right)+\sum_{E^{f} \in T_{H}^{p} \partial E^{p} \cap \Gamma_{N}^{p}} \mathbf{q} \cdot \mathbf{t}
\end{aligned}
$$

where the finite dimensional subspace $\mathbf{U}_{H} \subset \mathbf{U}\left(\Omega^{p}\right)$ is given by

$$
\mathbf{U}_{H}=\left\{\mathbf{q}=(u, v, w):\left.u\right|_{E^{p}},\left.v\right|_{E^{p}},\left.w\right|_{E^{p}} \in \mathbb{Q}_{1}\left(E^{p}\right) \forall E^{p} \in T_{H}^{p}, \mathbf{q}=0 \text { on } \Gamma_{D}^{p}\right\}
$$

The discrete displacements $\mathbf{u}_{H}$ and the corresponding strain tensor $\boldsymbol{\epsilon}\left(\mathbf{u}_{H}\right)$ are written in terms of the nodal displacement degrees of freedom represented by $\mathcal{U}$ as

$$
\begin{aligned}
& \mathbf{u}_{H}=\sum_{E^{p} \in T_{H}^{p}} \mathbf{N} \mathcal{U} \\
& \boldsymbol{\epsilon}\left(\mathbf{u}_{H}\right)=\sum_{E^{p} \in T_{H}^{p}} \mathbf{B} \mathcal{U}
\end{aligned}
$$


where $\mathbf{N}$ is the shape function matrix and $\mathbf{B}$ is the strain-displacement interpolation matrix. Equations (32), (33) and (34) eventually lead to the following system of equations

$$
\mathbf{K} \mathcal{U}=\mathbf{F}
$$

where $\mathbf{K}$ and $\mathbf{F}$ are refered to as the global stiffness matrix and global force vector respectively and are obtained as

$$
\begin{aligned}
& \mathbf{K}=\sum_{E^{p} \in T_{H}^{p}} \int_{E^{p}} \mathbf{B}^{T} \mathbb{D} \mathbf{B} \\
& \mathbf{F}=\sum_{E^{p} \in T_{H}^{p}} \int_{E^{p}} \mathbf{B}^{T} \alpha p \mathbf{I}+\sum_{E^{p} \in T_{H}^{p}} \int_{E^{p}} \mathbf{N}^{T}\left(\rho \phi \mathbf{g}+\rho_{r}(1-\phi) \mathbf{g}\right)+\sum_{E^{p} \in T_{H \partial E^{p} \cap \Gamma_{N}^{p}} \int_{N^{T}} \mathbf{N}^{T}}
\end{aligned}
$$

To simplify the computations, (35) is recast in compact engineering notation (see [32]) wherein stresses $\boldsymbol{\sigma}$, strains $\mathbf{\epsilon}$ and identity tensor $\mathbf{I}$ are represented as vectors and fourth order tensor $\mathbb{D}$ is represented as a second order tensor. The matrices $\mathbf{N}$ and $\mathbf{B}$ are also recast appropriately.

\subsection{Evaluation of integrals for the poromechanical solve using quadrature}

The trilinear function $F_{E}^{p}(\hat{\mathbf{r}}): \hat{\mathbf{r}} \mapsto \mathbf{r}^{p}$ which we have already defined in (14) can also be written as

$$
F_{E}^{p}(\hat{\mathbf{r}}): \hat{\mathbf{r}} \mapsto \mathbf{r}^{p}=\sum_{i=1}^{8} \hat{N}_{i}(\hat{\mathbf{r}}) \mathbf{r}_{i}^{p}
$$

where $\hat{N}_{i}(\hat{\mathbf{r}})$ are refered to as the shape functions and satisfy the property that $\hat{N}_{i}\left(\hat{\mathbf{r}}_{j}\right)=\delta_{i j}, i, j=1, \ldots, 8$ where $\delta_{i j}$ is the Kronecker delta, and are given as

$$
\begin{aligned}
& \hat{N}_{1}=(1-\hat{x})(1-\hat{y})(1-\hat{z}) \\
& \hat{N}_{2}=\hat{x}(1-\hat{y})(1-\hat{z}) \\
& \hat{N}_{3}=\hat{x} \hat{y}(1-\hat{z}) \\
& \hat{N}_{4}=(1-\hat{x}) \hat{y}(1-\hat{z}) \\
& \hat{N}_{5}=(1-\hat{x})(1-\hat{y}) \hat{z} \\
& \hat{N}_{6}=\hat{x}(1-\hat{y}) \hat{z} \\
& \hat{N}_{7}=\hat{x} \hat{y} \hat{z} \\
& \hat{N}_{8}=(1-\hat{x}) \hat{y} \hat{z}
\end{aligned}
$$

The shape function matrix is given as

$$
\begin{aligned}
\hat{\mathbf{N}} & =\left[\begin{array}{llllllll}
\overline{\mathbf{N}}_{1} & \overline{\mathbf{N}}_{2} & \overline{\mathbf{N}}_{3} & \overline{\mathbf{N}}_{4} & \overline{\mathbf{N}}_{5} & \overline{\mathbf{N}}_{6} & \overline{\mathbf{N}}_{7} & \overline{\mathbf{N}}_{8}
\end{array}\right]_{3 \times 24} \\
\overline{\mathbf{N}}_{i} & =\left[\begin{array}{ccc}
\hat{N}_{i} & 0 & 0 \\
0 & \hat{N}_{i} & 0 \\
0 & 0 & \hat{N}_{i}
\end{array}\right]_{3 \times 3}
\end{aligned}
$$

$(\forall i=1, . ., 8)$

The jacobian of $F_{E}^{p}(\hat{\mathbf{r}})$ is the given as

$$
D F_{E}^{p}(\hat{\mathbf{r}}) \equiv\left[\begin{array}{lll}
\frac{\partial x}{\partial \hat{x}} & \frac{\partial x}{\partial \hat{y}} & \frac{\partial x}{\partial \hat{z}} \\
\frac{\partial y}{\partial \hat{x}} & \frac{\partial y}{\partial \hat{y}} & \frac{\partial y}{\partial \hat{z}} \\
\frac{\partial z}{\partial \hat{x}} & \frac{\partial z}{\partial \hat{y}} & \frac{\partial z}{\partial \tilde{z}}
\end{array}\right]=\left[\begin{array}{cccc}
\sum_{i=1}^{8} \frac{\partial \hat{N}_{i}}{\partial \hat{x}} x_{i}^{p} & \sum_{i=1}^{8} \frac{\partial \hat{N}_{i}}{\partial \hat{y}} x_{i}^{p} & \sum_{i=1}^{8} \frac{\partial \hat{N}_{i}}{\partial \tilde{z}} x_{i}^{p} \\
\sum_{i=1}^{8} \frac{\partial \hat{N}_{i}}{\partial \hat{x}} y_{i}^{p} & \sum_{i=1}^{8} \frac{\partial \hat{N}_{i}}{\partial \hat{y}} y_{i}^{p} & \sum_{i=1}^{8} \frac{\partial \hat{N}_{i}}{\partial \tilde{z}} y_{i}^{p} \\
\sum_{i=1}^{8} \frac{\partial \hat{N}_{i}}{\partial \hat{x}} z_{i}^{p} & \sum_{i=1}^{8} \frac{\partial \hat{N}_{i}}{\partial \hat{y}} z_{i}^{p} & \sum_{i=1}^{8} \frac{\partial \hat{N}_{i}}{\partial \tilde{z}} z_{i}^{p}
\end{array}\right]
$$


The strain-displacement interpolation matrix is given as

$$
\begin{aligned}
& \hat{\mathbf{B}}=\left[\begin{array}{llllllll}
\hat{\mathbf{B}}_{1} & \hat{\mathbf{B}}_{2} & \hat{\mathbf{B}}_{3} & \hat{\mathbf{B}}_{4} & \hat{\mathbf{B}}_{5} & \hat{\mathbf{B}}_{6} & \hat{\mathbf{B}}_{7} & \hat{\mathbf{B}}_{8}
\end{array}\right]_{6 \times 24} \\
& \hat{\mathbf{B}}_{i}=\left[\begin{array}{ccc}
\frac{\partial \hat{\mathbf{N}}_{i}}{\partial x} & 0 & 0 \\
0 & \frac{\partial \hat{\mathbf{N}}_{i}}{\partial y} & 0 \\
0 & 0 & \frac{\partial \hat{\mathbf{N}}_{i}}{\partial z} \\
\frac{\partial \hat{\mathbf{N}}_{i}}{\partial y} & \frac{\partial \hat{\mathbf{N}}_{i}}{\partial x} & 0 \\
\frac{\partial \hat{\mathbf{N}}_{i}}{\partial z} & 0 & \frac{\partial \hat{\mathbf{N}}_{i}}{\partial x} \\
0 & \frac{\partial \hat{\mathbf{N}}_{i}}{\partial z} & \frac{\partial \hat{\mathbf{N}}_{i}}{\partial y}
\end{array}\right]_{6 \times 3}
\end{aligned}
$$

and the derivatives of the shape functions are obtained using

$$
\left[\begin{array}{l}
\frac{\partial \hat{\mathbf{N}}_{i}}{\partial x} \\
\frac{\partial \hat{\mathbf{N}}_{i}}{\partial y} \\
\frac{\partial \hat{\mathbf{N}}_{i}}{\partial z}
\end{array}\right]=\left(D F_{E}^{p}\right)^{-T}\left[\begin{array}{l}
\frac{\partial \hat{\mathbf{N}}_{i}}{\partial \hat{x}} \\
\frac{\partial \hat{\mathbf{N}}_{i}}{\partial \hat{y}} \\
\frac{\partial \mathbf{N}_{i}}{\partial \hat{z}}
\end{array}\right]=\left[\begin{array}{ccc}
\sum_{i=1}^{8} \frac{\partial \hat{N}_{i}}{\partial \hat{x}} x_{i}^{p} & \sum_{i=1}^{8} \frac{\partial \hat{N}_{i}}{\partial \hat{y}} x_{i}^{p} & \sum_{i=1}^{8} \frac{\partial \hat{N}_{i}}{\partial \hat{z}} x_{i}^{p} \\
\sum_{i=1}^{8} \frac{\partial \hat{N}_{i}}{\partial \hat{x}} y_{i}^{p} & \sum_{i=1}^{8} \frac{\partial \hat{N}_{i}}{\partial \hat{y}} y_{i}^{p} & \sum_{i=1}^{8} \frac{\partial \hat{N}_{i}}{\partial \hat{z}} y_{i}^{p} \\
\sum_{i=1}^{8} \frac{\partial \hat{N}_{i}}{\partial \hat{x}} z_{i}^{p} & \sum_{i=1}^{8} \frac{\partial \hat{N}_{i}}{\partial \hat{y}} z_{i}^{p} & \sum_{i=1}^{8} \frac{\partial \hat{N}_{i}}{\partial \hat{z}} z_{i}^{p}
\end{array}\right]^{-T}\left[\begin{array}{c}
\frac{\partial \hat{\mathbf{N}}_{i}}{\partial \hat{x}} \\
\frac{\partial \hat{\mathbf{N}}_{i}}{\partial \hat{y}} \\
\frac{\partial \hat{\mathbf{N}}_{i}}{\partial \hat{z}}
\end{array}\right]
$$

The elasticity tensor for the isotropic porous solid is

$$
\mathbb{D}=\left[\begin{array}{cccccc}
\lambda+2 G & \lambda & \lambda & 0 & 0 & 0 \\
\lambda & \lambda+2 G & \lambda & 0 & 0 & 0 \\
\lambda & \lambda & \lambda+2 G & 0 & 0 & 0 \\
0 & 0 & 0 & G & 0 & 0 \\
0 & 0 & 0 & 0 & G & 0 \\
0 & 0 & 0 & 0 & 0 & G
\end{array}\right]_{6 \times 6}
$$

where $\lambda$ is the Lame parameter corresponding to the drained response of the porous specimen and $G$ is the shear modulus of porous specimen. Denoting the determinant of $D F_{E}^{p}(\hat{\mathbf{r}})$ by $J_{E}^{p}$, the integrals leading upto the element stiffness matrix and force vectors are given as

$$
\left.\begin{array}{c}
\left.\int_{E^{p}} \mathbf{N}^{T} \mathbf{f} \equiv \int_{\hat{E}} \hat{\mathbf{N}}^{T} \mathbf{f} J_{E}^{p} \equiv \sum_{g \in G}\left(\hat{\mathbf{N}}^{T} \mathbf{f} J_{E}^{p}\right)\right|_{g} w_{g} \\
\left.\int_{E^{p}} \mathbf{B}^{T} \alpha p \mathbf{I} \equiv \int_{\hat{E}} \hat{\mathbf{B}}^{T} \alpha p \mathbf{I} J_{E}^{p} \equiv \sum_{g \in G}\left(\hat{\mathbf{B}}^{T} \alpha p \mathbf{I} J_{E}^{p}\right)\right|_{g} w_{g} \\
\left.\int_{E^{p}} \mathbf{B}^{T} \mathbb{D} \mathbf{B} \equiv \int_{\hat{E}} \hat{\mathbf{B}}^{T} \mathbb{D} \hat{\mathbf{B}} J_{E}^{p} \equiv \sum_{g \in G}\left(\hat{\mathbf{B}}^{T} \mathbb{D} \hat{\mathbf{B}} J_{E}^{p}\right)\right|_{g} w_{g} \\
\left.\int_{\partial E^{p} \cap \Gamma_{N}^{p}} \mathbf{N}^{T} \mathbf{t} \equiv \int_{\partial \hat{E} \cap \Gamma_{N}^{p}} \hat{\mathbf{N}}^{T} \mathbf{t} J_{E}^{p} \equiv \sum_{g \in G \cap \partial \hat{E}}\left(\hat{\mathbf{N}}^{T} \mathbf{t} J_{E}^{p}\right)\right|_{g} w_{g}
\end{array}\right\}
$$

where $G$ is the set (of cardinality 8 ) of quadrature points, $\left.(\cdot)\right|_{g}$ is the quantity $(\cdot)$ evaluated at the quadrature point $g \in G, \mathbf{I}=\left[\begin{array}{llllll}1 & 1 & 1 & 0 & 0 & 0\end{array}\right]^{T}$ and $w_{g}$ is the weight associated with a quadrature point $g \in G$. In lieu of (35)-(37) and (38), we write

$$
\mathbf{K U}=\mathbf{F}
$$


where the global stiffness matrix $\mathbf{K}$ and global force vector $\mathbf{F}$

$$
\begin{aligned}
\mathbf{K}= & \left.\sum_{E^{p} \in T_{H}^{p}} \sum_{g \in G}\left(\hat{\mathbf{B}}^{T} \mathbb{D} \hat{\mathbf{B}} J_{E}^{p}\right)\right|_{g} w_{g} \\
\mathbf{F}= & \left.\sum_{E^{p} \in T_{H}^{p}} \sum_{g \in G}\left(\hat{\mathbf{B}}^{T} \alpha p \mathbf{I} J_{E}^{p}\right)\right|_{g} w_{g}+\left.\sum_{E^{p} \in T_{H}^{p}} \sum_{g \in G}\left(\hat{\mathbf{N}}^{T} \mathbf{f} J_{E}^{p}\right)\right|_{g} w_{g} \\
& +\left.\sum_{E^{p} \in T_{H}^{p}} \sum_{g \in G \cap \partial \hat{E}}\left(\hat{\mathbf{N}}^{T} \mathbf{t} J_{E}^{p}\right)\right|_{g} w_{g}
\end{aligned}
$$

are obtained by assembling the element stiffness matrix and element force vectors. The reader is refered to [33] for the details of the assembly process. The set $G$ of quadrature points with associated weights is

$$
\begin{aligned}
& g_{1} \equiv\left(\frac{1}{2}\left(1-\frac{\sqrt{3}}{3}\right), \frac{1}{2}\left(1-\frac{\sqrt{3}}{3}\right), \frac{1}{2}\left(1-\frac{\sqrt{3}}{3}\right)\right) \\
& g_{2} \equiv\left(\frac{1}{2}\left(1+\frac{\sqrt{3}}{3}\right), \frac{1}{2}\left(1-\frac{\sqrt{3}}{3}\right), \frac{1}{2}\left(1-\frac{\sqrt{3}}{3}\right)\right) \\
& g_{3} \equiv\left(\frac{1}{2}\left(1+\frac{\sqrt{3}}{3}\right), \frac{1}{2}\left(1+\frac{\sqrt{3}}{3}\right), \frac{1}{2}\left(1-\frac{\sqrt{3}}{3}\right)\right) \\
& g_{4} \equiv\left(\frac{1}{2}\left(1-\frac{\sqrt{3}}{3}\right), \frac{1}{2}\left(1+\frac{\sqrt{3}}{3}\right), \frac{1}{2}\left(1-\frac{\sqrt{3}}{3}\right)\right) \\
& g_{5} \equiv\left(\frac{1}{2}\left(1-\frac{\sqrt{3}}{3}\right), \frac{1}{2}\left(1-\frac{\sqrt{3}}{3}\right), \frac{1}{2}\left(1+\frac{\sqrt{3}}{3}\right)\right) \\
& g_{6} \equiv\left(\frac{1}{2}\left(1+\frac{\sqrt{3}}{3}\right), \frac{1}{2}\left(1-\frac{\sqrt{3}}{3}\right), \frac{1}{2}\left(1+\frac{\sqrt{3}}{3}\right)\right) \\
& g_{7} \equiv\left(\frac{1}{2}\left(1+\frac{\sqrt{3}}{3}\right), \frac{1}{2}\left(1+\frac{\sqrt{3}}{3}\right), \frac{1}{2}\left(1+\frac{\sqrt{3}}{3}\right)\right) \\
& g_{8} \equiv\left(\frac{1}{2}\left(1-\frac{\sqrt{3}}{3}\right), \frac{1}{2}\left(1+\frac{\sqrt{3}}{3}\right), \frac{1}{2}\left(1+\frac{\sqrt{3}}{3}\right)\right) \\
& w_{g}=\frac{1}{8} \quad \forall g \in G
\end{aligned}
$$

\subsection{Lagrangian porosity update during the flow solve with the fixed mean stress constraint}

The Eulerian porosity variation in a deformable porous medium is approximated as ([34], [35])

$$
\delta \phi=\left(\frac{\alpha-\phi}{K_{b}}\right)\left(\delta \sigma_{v}+\delta p\right)
$$

Imposing the fixed mean stress constraint $\delta \sigma_{v}=0$ in (42) results in

$$
\delta \phi=\left(\frac{\alpha-\phi}{K_{b}}\right) \delta p
$$

Using the relation for the mean stress $\sigma_{v}=K_{b} \bar{\epsilon}-\alpha p$ and the fixed mean stress constraint $\delta \sigma_{v}=0$ results in

$$
\delta \bar{\epsilon}=\frac{\alpha}{K_{b}} \delta p
$$


which implies that the evolution law for volumetric strain is given by

$$
\epsilon^{k+1}=\epsilon^{k}+\frac{\alpha}{K_{b}} \delta^{(k)} p
$$

In lieu of (43) and the relationship $\phi^{*}=\phi(1+\bar{\epsilon})$, the Lagrangian porosity variation is given by

$$
\delta \phi^{*}=\phi \delta \bar{\epsilon}+(1+\bar{\epsilon}) \delta \phi=\left((\phi+1+\bar{\epsilon}) \frac{\alpha}{K_{b}}-\frac{\phi^{*}}{K}\right) \delta p
$$

which implies that the evolution law for Lagrangian porosity is given by

$$
\phi^{*^{k+1}}=\phi^{* k}+\overbrace{\left(\left(\phi^{k}+1+\bar{\epsilon}^{k}\right) \frac{\alpha}{K_{b}}-\frac{\phi^{*^{k}}}{K_{b}}\right)}^{\left.\frac{\partial \phi^{*}}{\partial p}\right|_{k}} \delta^{(k)} p
$$

\subsection{Lagrangian porosity update during the poromechanical solve}

Invoking (42) again and using the relation $\delta \sigma_{v}=K_{b} \delta \bar{\epsilon}-\alpha \delta p$, we get

$$
\delta \phi=(\alpha-\phi) \delta \bar{\epsilon}+\frac{(\alpha-\phi)(1-\alpha)}{K_{b}} \delta p
$$

In lieu of (45) and the relationship $\phi^{*}=\phi(1+\bar{\epsilon})$, the Lagrangian porosity variation is given by

$$
\begin{aligned}
& \delta \phi^{*}=\phi \delta \bar{\epsilon}+(1+\bar{\epsilon}) \delta \phi=\phi \delta \bar{\epsilon}+(1+\bar{\epsilon})\left((\alpha-\phi) \delta \bar{\epsilon}+\frac{(\alpha-\phi)(1-\alpha)}{K_{b}} \delta p\right) \\
& =(\alpha+\bar{\epsilon}(\alpha-\phi)) \delta \bar{\epsilon}+(1+\bar{\epsilon}) \frac{(\alpha-\phi)(1-\alpha)}{K_{b}} \delta p
\end{aligned}
$$

Following the arguments of [25], linear poroelasticity consists in setting the tangents $(\alpha+\bar{\epsilon}(\alpha-\phi))$ and $(1+$ $\bar{\epsilon}) \frac{(\alpha-\phi)(1-\alpha)}{K_{b}}$ in (46) as constants. Hence (46) is rewritten as

$$
\delta \phi^{*}=\left(\alpha+\bar{\epsilon}_{0}\left(\alpha-\phi_{0}\right)\right) \delta \bar{\epsilon}+\left(1+\bar{\epsilon}_{0}\right) \frac{\left(\alpha-\phi_{0}\right)(1-\alpha)}{K_{b}} \delta p
$$

which can be integrated from initial time to obtain

$$
\phi^{*^{m}}=\phi_{0}+\left(\alpha+\bar{\epsilon}_{0}\left(\alpha-\phi_{0}\right)\right)\left(\bar{\epsilon}^{m}-\bar{\epsilon}_{0}\right)+\left(1+\bar{\epsilon}_{0}\right) \frac{\left(\alpha-\phi_{0}\right)(1-\alpha)}{K_{b}}\left(p^{m}-p_{0}\right)
$$

where $(\cdot)^{m}$ refers to $(\cdot)$ evaluated at the $m^{t h}$ coupling iteration. In lieu of the small strain assumption (9), the $O\left(\epsilon^{2}\right)$ terms are neglected to obtain

$$
{\phi^{*}}^{*^{m}}=\phi_{0}+\alpha\left(\bar{\epsilon}^{m}-\bar{\epsilon}_{0}\right)+\left(1+\bar{\epsilon}_{0}\right) \frac{\left(\alpha-\phi_{0}\right)(1-\alpha)}{K_{b}}\left(p^{m}-p_{0}\right)
$$

which, under the initial condition (11) lending to $\bar{\epsilon}_{0}=0$, is finally written as

$$
\phi^{*^{m}}=\phi_{0}+\alpha \bar{\epsilon}^{m}+\frac{\left(\alpha-\phi_{0}\right)(1-\alpha)}{K_{b}}\left(p^{m}-p_{0}\right)
$$




\section{THE TWO-GRID STAGGERED SOLUTION ALGORITHM}

The flowchart of the algorithm is provided in Figure 4. The algorithm solves the flow system on a fine mesh while freezing the mean stress of the porous solid followed by the poromechanics system on a coarse mesh. The pressures at the end of the flow solve along with updated fluid densities and Lagrangian porosities are upscaled and these upscaled quantities contribute to the force vector in the poromechanics system. The poromechanics system is then solved for nodal displacements which are post-processed to obtain volumetric strains. These volumetric strains along with upscaled pressures are then used to update the Lagrangian porosities in the poromechanical grid. These coarse grid Lagrangian porosities are then downscaled onto the flow grid. This two-step procedure of nonlinear flow solve followed by linear poromechanics solve is continued until a convergence criterion (evaluated at the coarse scale poromechanics grid) is met. Once this criterion is met, we march forward to the next time step as shown in Algorithm 1.

\subsection{Convergence criterion for coupling iterations}

The stopping criterion for coupling iterations is designed based on the requirement that the change in mean stress across coupling iterations in each time step reduces monotonically from one coupling iteration to the next. We already know that one coupling iteration consists of one flow solve and one poromechanics solve. We also know that the fixed stress split strategy imposes a fixed mean stress during the flow solve in every coupling iteration. This implies that the mean stress only changes during the poromechanics solve in every coupling iteration. This automatically implies that the change in mean stress across one coupling iteration is identical to the change in mean stress during the poromechanics solve in that coupling iteration. As a result, the stopping criterion is to be designed based on the requirement that the change in mean stress across poromechanics solves reduces monotonically from one coupling iteration to the next. Now, let us look at the expression (42) for porosity variation in deformable porous medium given by

$$
\delta \phi=\left(\frac{\alpha-\phi}{K_{b}}\right)\left(\delta \sigma_{v}+\delta p\right)
$$

and let us write it in two forms

$$
\begin{aligned}
& \left.(\delta \phi)\right|_{f}=\left(\frac{\alpha-\phi}{K_{b}}\right)\left(\left.\left(\delta \sigma_{v}\right)\right|_{f}+\left.(\delta p)\right|_{f}\right) \\
& \left.(\delta \phi)\right|_{c}=\left(\frac{\alpha-\phi}{K_{b}}\right)\left(\left.\left(\delta \sigma_{v}\right)\right|_{c}+\left.(\delta p)\right|_{c}\right)
\end{aligned}
$$

where $\left.\delta(\cdot)\right|_{f}$ is the change in quantity (.) during the flow solve in a coupling iteration and $\left.\delta(\cdot)\right|_{c}$ is the change in quantity $(\cdot)$ over the entire coupling iteration. Now the fixed mean stress constraint implies that $\left.\left(\delta \sigma_{v}\right)\right|_{f}=0$ and since the pressure does not change during the poromechanics solve, $\left.(\delta p)\right|_{f}=\left.(\delta p)\right|_{c}$. As a result, we have

$$
\left.(\delta \phi)\right|_{c}-\left.(\delta \phi)\right|_{f}=\left.\left.\left(\frac{\alpha-\phi}{K_{b}}\right)\left(\delta \sigma_{v}\right)\right|_{c} \equiv\left(\frac{\alpha-\phi}{K_{b}}\right)\left(\delta \sigma_{v}\right)\right|_{m}
$$

where $\left.\left(\delta \sigma_{v}\right)\right|_{m}$ is the change in mean stress during the poromechanics solve in the coupling iteration. In lieu of the above, the stopping criterion is designed based on the change in porosity during the poromechanics solve in every coupling iteration. Since we calculate the Lagrangian porosity updates after every flow and poromechanics solve, the stopping criterion is based in the requirement that the change in Lagrangian porosity during the poromechanics solve in every coupling iteration reduces monotonically from one coupling iteration to the next. Now the change in Lagrangian porosity during the poromechanics solve in the $m^{t h}$ coupling iteration in every time step is given by

$$
\phi^{*^{m}}-\phi^{*^{k+1, m}}
$$


where $\phi^{*^{m}}$ is the Lagrangian porosity at the end of the $m^{t h}$ coupling iteration, $\phi^{*^{k+1, m}}$ is the Lagrangian porosity at the end of the flow solve in the $m^{\text {th }}$ coupling iteration and $k+1$ is the number of Newton iterations required to solve the nonlinear flow problem. This quantity is normalized by the current value of Lagrangian porosity i.e. $\phi^{*^{m}}$ as follows

$$
\frac{\phi^{*^{m}}-\phi^{*^{k+1, m}}}{\phi^{*^{m}}}
$$

and the stopping criterion is based on the requirement that the maximum norm of this quantity is less than a certain pre-specified tolerance as follows

$$
\left\|\frac{\phi^{*^{m}}-\phi^{*^{k+1, m}}}{\phi^{*^{m}}}\right\|_{\infty} \leq \mathrm{TOL}
$$

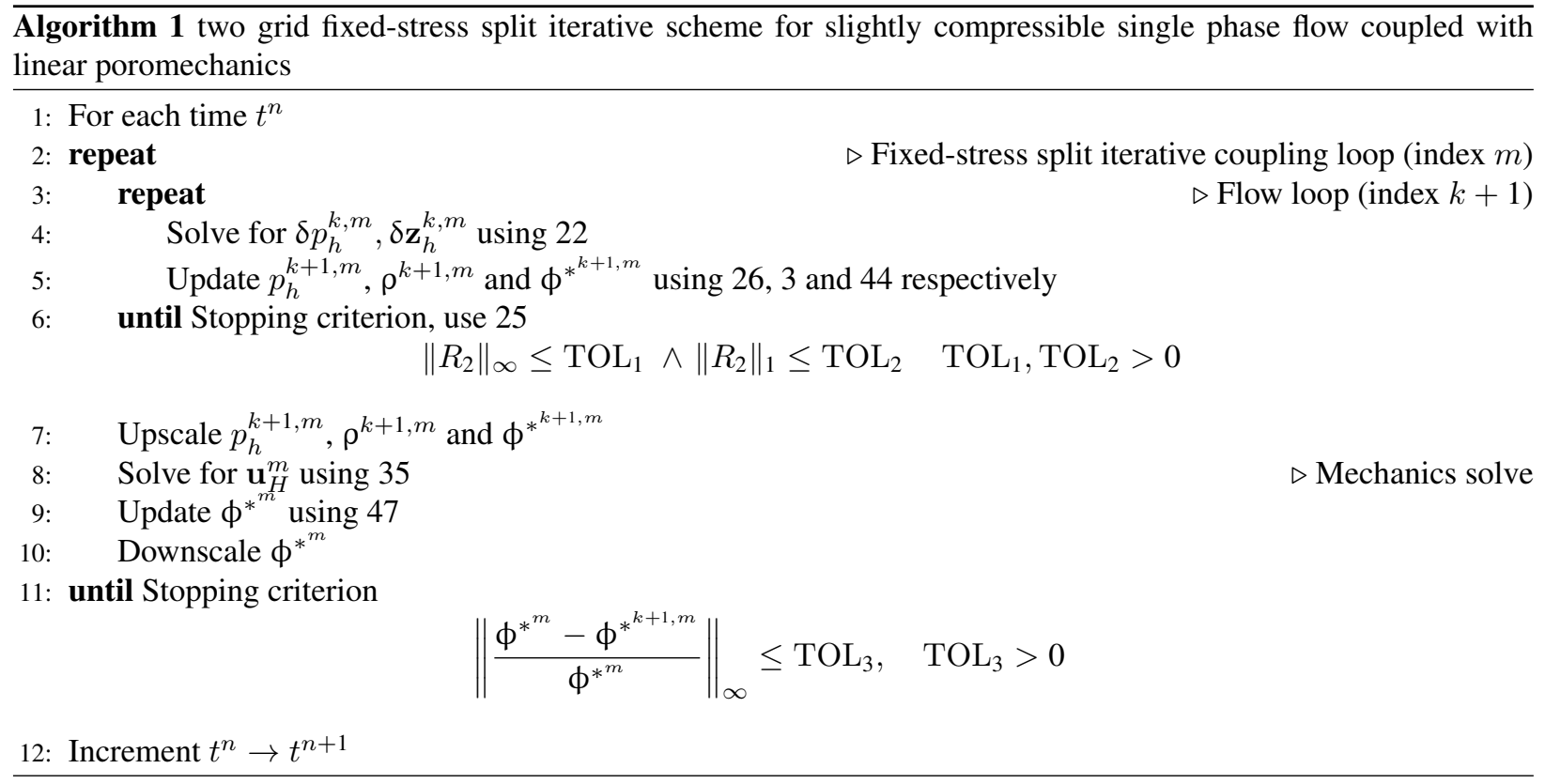

\section{SUMMARY}

We present the model equations for slightly compressible single phase flow coupled with linear poromechanics for flow through porous media. We then present the discrete variational statements for the two-grid staggered solution algorithm. The two-grid algorithm connotates that the model equations for flow and poromechanics are solved on different finite element grids. Flow is solved on a fine mesh using a multipoint flux mixed finite element method and poromechanics is solved on a coarse mesh using a conforming Galerkin finite element method. The notion of staggering lies in solving the flow and poromechanics equations sequentially in a coupling iteration instead of solving the coupled system of equations monolithically. The system solve at a particular time step can consist of multiple coupling iterations depending on when the stopping criterion is achieved. In essence, the staggering imposes a constraint on the system during the flow solve thereby working as a predictor to the state of the system followed by the poromechanics solve which works as a corrector to the state of the system at the end of the coupling iteration. 


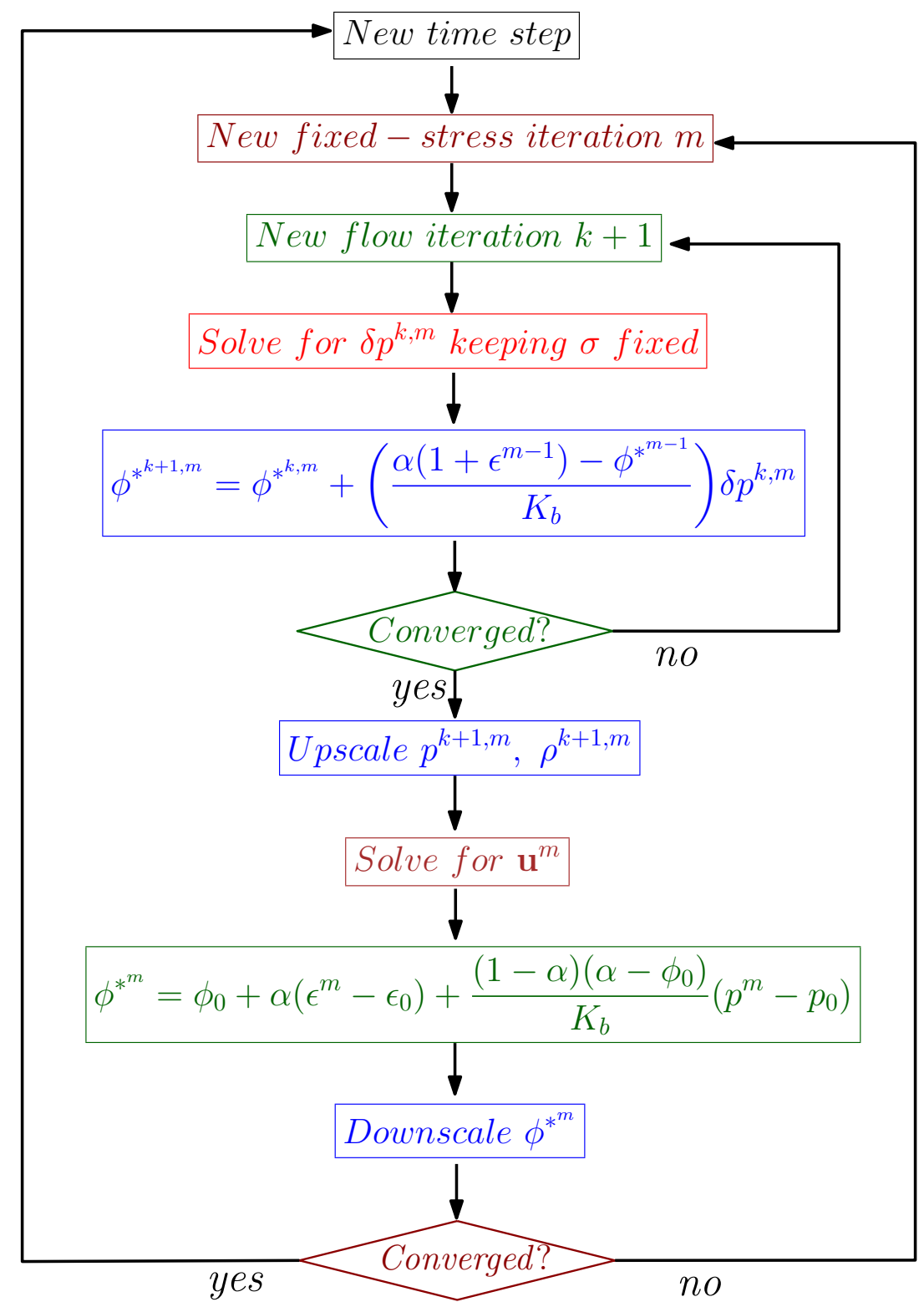

FIG. 4: Flowchart for two grid fixed-stress split iterative scheme for single phase flow coupled with linear poromechanics and $(\cdot)^{k, m}$ denotes the quantity $(\cdot)$ evaluated at the $k^{t h}$ flow iteration within the $m^{t h}$ coupling iteration and $(\cdot)^{m}$ denotes the quantity $(\cdot)$ evaluated at the $m^{\text {th }}$ coupling iteration.

\section{PARALLELISM INVOLVED}

The elements of the geomechanical domain are first divided areally $(y-z)$ among MPI processes. Since the flow domain is a subset of the geomechanical domain, the areal extent of the flow domain would be smaller than that of the geomechanical domain. In lieu of that, not all processes would occupy elements of the flow domain. We need to identify the processes that would occupy flow elements, and then carve out a MPI subcommunicator with only those processes involved. This subcommunicator would be involved in all the tasks to be performed in order to solve the flow problem in parallel. For example, with reference to Figure 5, let us assume that we decide to work with 15 
processes. The default MPI communicator would be

$$
\text { mpi_comm_world }=\{0,1,2,3,4,5,6,7,8,9,10,11,12,13,14\}
$$

Thereafter, based on the actual location of the areal $(y-z)$ boundaries of the flow domain, the flow elements are
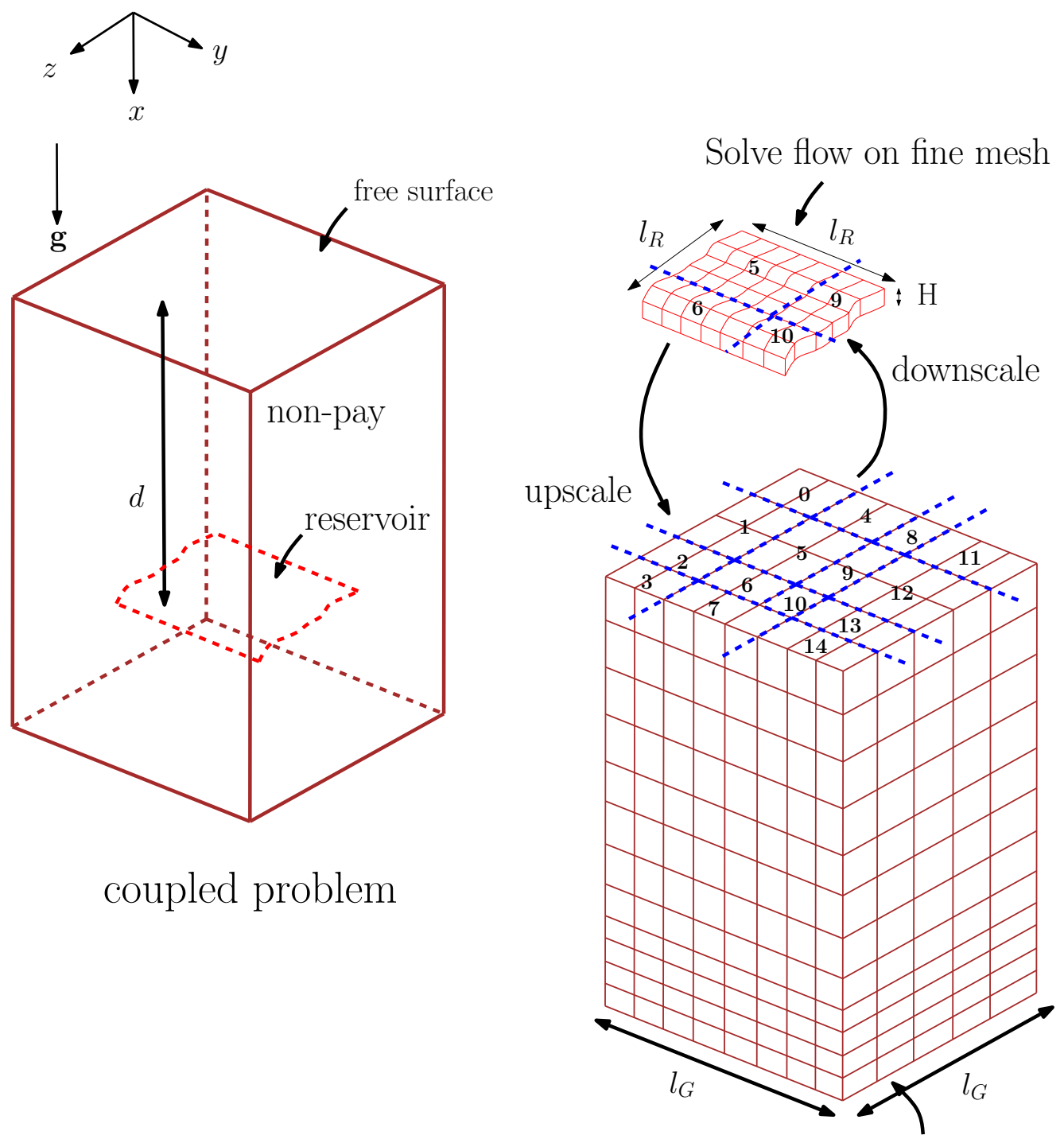

Solve mechanics on coarse mesh

FIG. 5: The numbers are process ranks, and the division of elements among processes is areal. For this particular case, the default MPI communicator has 15 processes. Since the flow domain is a subset of the geomechanical domain, not all processes would occupy flow elements. The MPI subcommunicator would have only those processes that occupy flow elements. Only the processes involved in the subcommunicator would be involved in the construction of grid-to-grid projection operators.

assigned to processes based on the areal boundaries of the processes. With reference to Figure 5, processes with ranks $5,6,9,10$ occupy elements of the flow domain. Thus, the subcommunicator would be

$$
\text { flow_comm }=\{5,6,9,10\}
$$


This means that processes with ranks $0,1,2,3,4,7,8,11,12,13$, 14 do not occupy flow elements, and need not participate in construction of grid-to-grid projection operators. The non-participation of as many as 11 processes in the construction of projection operators, and indeed the flow solve, substantially reduces the memory load on the supercomputer. Data structures (with concomitant memory allocation) required for the flow solve need only be assigned to 4 processes instead of the usual 15 for this particular case.

\section{IDENTIFYING PAIRS THAT INTERSECT WITH ONE ANOTHER}

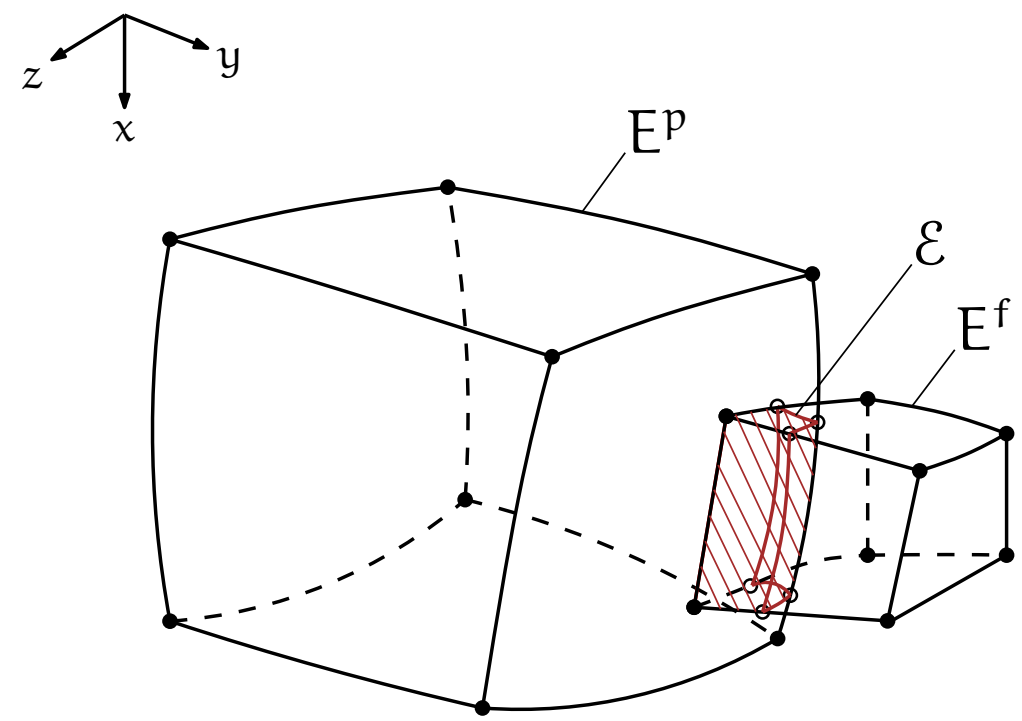

FIG. 6: Construction of global up and down scaling operators are designed around the assembly of local up and down scaling operators.

The global upscaling and downscaling operators are constructed in the pre-processing step by applying a local mapping procedure to each pair of overlapping finite elements $E^{p}$ and $E^{f}$ of the two grids. As shown in Figure 6, we denote the intersection polyhedron of $E^{p}$ and $E^{f}$ by $\mathcal{E}$, and further denote the partitions of $E^{p}$ and $E^{f}$ by $\mathcal{I}^{E^{p}}$ and $\mathcal{I}^{E^{f}}$ respectively such that

$$
\begin{array}{lll}
E^{p}=\bigcup_{\mathcal{E} \in \mathcal{I}^{E^{p}}} \mathcal{E} & \text { where } & \mathcal{I}^{E^{p}} \equiv\left\{\mathcal{E}: \mathcal{E} \equiv E^{p} \cap E^{f} \forall E^{f} \in \mathcal{T}_{h}^{f}\right\} \\
E^{f}=\bigcup_{\mathcal{E} \in \mathcal{I}^{E^{f}}} \mathcal{E} & \text { where } & \mathcal{I}^{E^{f}}=\left\{\mathcal{E}: \mathcal{E}=E^{f} \cap E^{p} \forall E^{p} \in \mathcal{T}_{H}^{p}\right\}
\end{array}
$$

We first need to identify pairs of flow and poromechanics elements that intersect one another. Each pair will have one flow element and one poromechanics element. To determine whether a flow element is geometrically disjoint to a poromechanics element, we need to design an algorithm with all 8 vertices of the flow element. If all vertices of the flow element are inside the poromechanics element, then the flow element is inside the poromechanics element. If all vertices of the flow element are outside the poromechanics element, then the flow element is geometrically disjoint to the poromechanics element. If neither of the above two cases is true, then the flow element intersects the poromechanics element. A graphic description of the algorithm to determine if a point is inside or outside a finite element is given in Figure 7. The algorithm itself is given in Algorithm 2. 

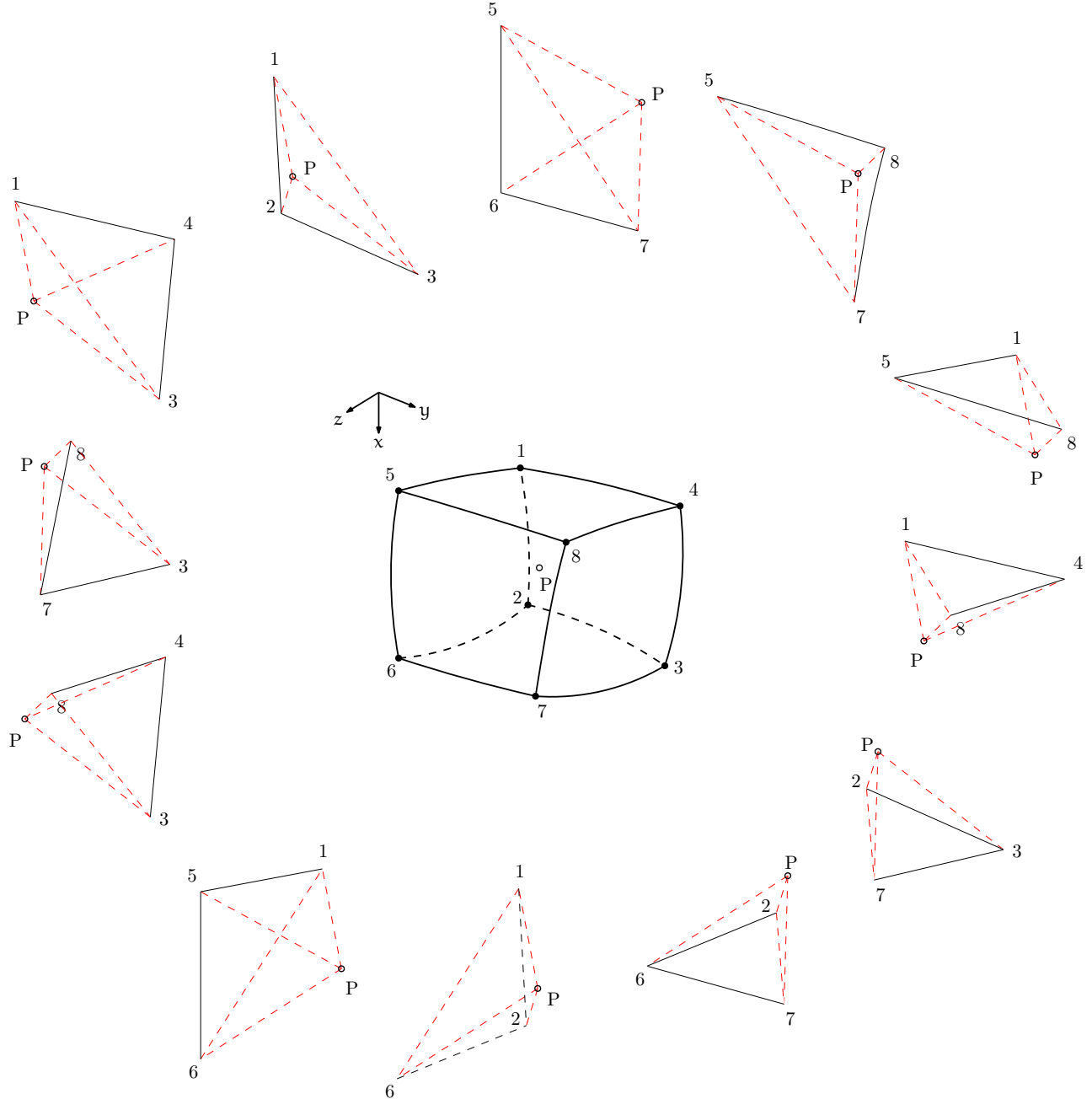

FIG. 7: Determing whether point $P$ is inside or outside the finite element 1-2-3-4-5-6-7-8. If point $P$ is inside the finite element, the sum of volumes of the 12 tetrahedra formed by $\mathrm{P}$ with the vertices of the finite element would be roughly equal to volume of the finite element. If point $\mathrm{P}$ is outside the finite element, the sum of volumes of the 12 tetrahedra would be greater than the volume of the finite element

\section{UPSCALING PORE PRESSURE AND BULK DENSITY FOR EACH PAIR}

Let $p^{E^{f}}$ and $\rho^{E^{f}}$ represent the piecewise constant pore pressure and fluid density respectively at $E^{f}$ such that

$$
p^{\mathcal{E}}=p^{E^{f}}, \quad \rho^{\mathcal{E}}=\rho^{E^{f}}
$$

The pore pressures are upscaled via the force vector term $\int_{E^{p}} \mathbf{B}^{T} \alpha p \mathbf{I}$ in (37) as follows

$$
\int_{E^{p}} \mathbf{B}^{T} \alpha p \mathbf{I}=\int_{E^{p}} \mathbf{B}^{T} \alpha\left(\sum_{\mathcal{E} \in \mathcal{I}^{E^{p}}} \frac{\operatorname{Meas}(\mathcal{E})}{\operatorname{Meas}\left(E^{p}\right)} p^{\mathcal{E}}\right) \mathbf{I}
$$




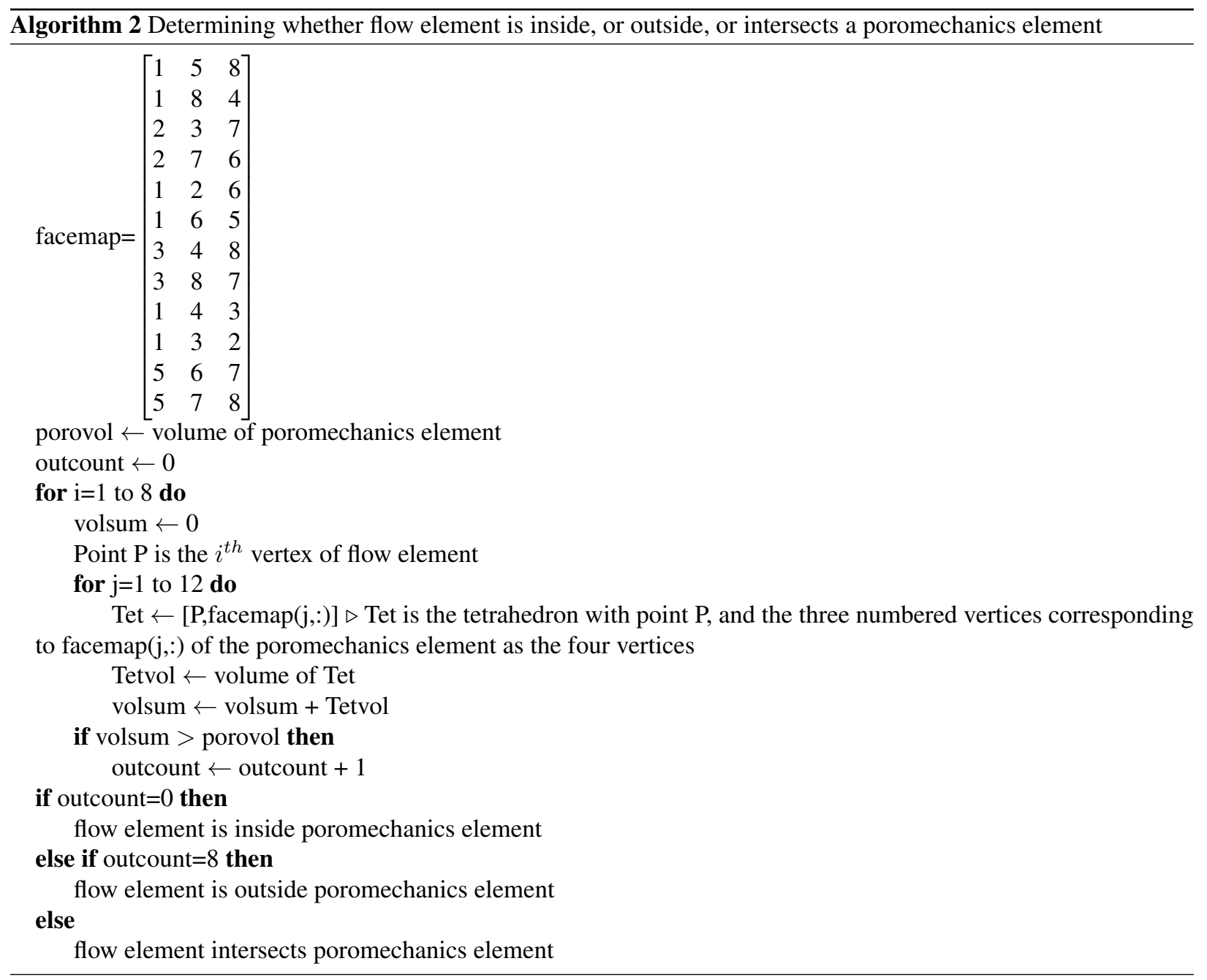

The bulk densities are upscaled via the force vector term $\int_{E^{p}} \mathbf{N}^{T} \rho \phi \mathrm{g}$ in (37) as follows

$$
\int_{E^{p}} \mathbf{N}^{T} \rho \phi \mathbf{g}=\int_{E^{p}} \mathbf{N}^{T}\left(\sum_{\mathcal{E} \in \mathcal{I}^{E^{p}}} \frac{\operatorname{Meas}(\mathcal{E})}{\operatorname{Meas}\left(E^{p}\right)} \rho^{\mathcal{E}} \phi^{\mathcal{E}}\right) \mathbf{g}
$$

In essence, the upscaled pore pressures and bulk densities on $E^{p}$ are local volume averages over $\mathcal{I}^{E^{p}}$ of the information obtained after the flow solve. The reason for choosing the local volume average approach for upscaling of the fine scale pore pressure and product of fine scale fluid density and porosity is the following: Suppose $v$ is any variable being projected onto a space of constants $W_{H} \equiv \mathbb{P}_{0}\left(T_{H}^{p}\right)$ defined on $T_{H}^{p}$. Then, the Galerkin orthogonal projection of $v$ onto $W_{H}$ would be

$$
\int_{\Omega^{p}}(v-\mathcal{R} v) w=0 \quad \forall w \in W_{H}
$$


where $\mathcal{R} v \in W_{H}$ is the projected quantity. Let $v^{\prime}$ denote the restriction of $v$ to $E^{p}$ and let $w^{\prime}$ denote the restriction of $w$ to $E^{p}$. We rewrite (50) as

$$
\sum_{E^{p} \in \mathcal{T}_{H}^{p}} \int_{E^{p}}\left(v^{\prime}-\mathcal{R} v\right) w^{\prime}=0
$$

which, in lieu of the linear independence of the basis $w^{\prime}$ of the space $W_{H}$, implies that

$$
\mathcal{R} v=\frac{1}{\operatorname{Meas}\left(E^{p}\right)} \int_{E^{p}} v^{\prime} \quad\left(\forall E^{p} \in T_{H}^{p}\right)
$$

If $v^{\prime}$ is defined by the discontinuous piecewise constants $v^{\mathcal{E}}$ on $\mathcal{I}^{E^{p}}\left(\forall E^{p} \in T_{H}^{p}\right)$, we get

$$
\int_{E^{p}} v^{\prime} \equiv \sum_{\mathcal{E} \in \mathcal{I}^{E^{p}}} \int_{\mathcal{E}} v^{\mathcal{E}}=\sum_{\mathcal{E} \in \mathcal{I}^{E^{p}}} v^{\mathcal{E}} \operatorname{Meas}(\mathcal{E}) \quad\left(\forall E^{p} \in T_{H}^{p}\right)
$$

In lieu of (51) and (52), we get

$$
\mathcal{R} v=\frac{1}{\operatorname{Meas}\left(E^{p}\right)} \sum_{\mathcal{E} \in \mathcal{I}^{E^{p}}} v^{\mathcal{E}} \operatorname{Meas}(\mathcal{E}) \equiv \sum_{\mathcal{E} \in \mathcal{I}^{E^{p}}} \frac{\operatorname{Meas}(\mathcal{E})}{\operatorname{Meas}\left(E^{p}\right)} v^{\mathcal{E}} \quad\left(\forall E^{p} \in T_{H}^{p}\right)
$$

\section{DOWNSCALING LAGRANGIAN POROSITY FOR EACH PAIR}

Let $W_{h} \equiv \mathbb{P}_{0}\left(T_{h}^{f}\right)$ represent the space of constants defined on $T_{h}^{f}$. Let $\mathcal{P} \phi^{*} \in W_{h}$ be the prolongation of the coarse scale Lagrangian porosity $\phi^{*}\left(\mathbf{x}^{p}\right), \mathbf{x}^{p} \in \Omega^{p} \cap \Omega^{f}$ onto $W_{h}$. Define $\mathcal{P}$ by

$$
\int_{\Omega^{f}}\left(\phi^{*}(\mathbf{x})-\left(\mathcal{P} \phi^{*}\right)(\mathbf{x})\right) w=0 \quad \forall w \in W_{h}
$$

Let $\phi^{\prime}\left(\mathbf{x}^{\prime}\right), \mathbf{x}^{\prime} \in E^{f}$ denote the restriction of $\phi^{*}(\mathbf{x})$ to $E^{f}$ and let $w^{\prime}$ denote the restriction of $w$ to $E^{f}$. We rewrite (53) as

$$
\sum_{E^{f} \in \mathcal{T}_{h}^{f}} \int_{E^{f}}\left(\phi^{\prime}\left(\mathbf{x}^{\prime}\right)-\mathcal{P} \phi^{*}\right) w^{\prime}=0
$$

which, in lieu of the linear independence of the basis $w^{\prime}$ of the space $W_{h}$, implies that

$$
\mathcal{P} \phi^{*}=\frac{1}{\operatorname{Meas}\left(E^{f}\right)} \int_{E^{f}} \phi^{\prime}\left(\mathbf{x}^{\prime}\right) \quad\left(\forall E^{f} \in T_{h}^{f}\right)
$$

Let $\phi^{*^{E^{p}}}$ be the piecewise constant Lagrangian porosity at $E^{p}\left(\forall E^{p} \in T_{H}^{p}\right)$ such that

$$
\phi^{*^{\mathcal{E}}}=\phi^{*^{E^{p}}}
$$

Then $\phi^{\prime}\left(\mathbf{x}^{\prime}\right)$ is defined by discontinuous piecewise constants over $\mathcal{I}^{E^{f}}$ as

$$
\phi^{\prime}\left(\mathbf{x}^{\prime}\right)=\phi^{*^{\mathcal{E}}}=\phi^{*^{E^{p}}}
$$

$\left(\forall \mathbf{x}^{\prime} \in \mathcal{E}\right.$ and $\left.\mathcal{E} \in E^{p}\right)$

In lieu of the above, we get

$$
\mathcal{P} \phi^{*}=\frac{\sum_{\mathcal{E} \in \mathcal{I}^{E^{f}}} \phi^{*^{E^{p}}} \operatorname{Meas}(\mathcal{E})}{\operatorname{Meas}\left(E^{f}\right)}
$$

$\left(\mathcal{E}=E^{f} \cap E^{p}\right)$ 


\section{LOCAL OPERATORS : IMPLEMENTATION}

$\checkmark$ The first step is to obtain the equations of the faces of $E^{p}$ and $E^{f}$ as explained in Section 11.1.

$\checkmark$ The next step is to design an algorithm that uses the equations of the element faces to obtain points on the periphery of the intersection polyhedron as explained in Section 11.2.

$\checkmark$ The final step is to use the set of points obtained on the periphery of the intersection polyhedron to determine its measure as explained in Section 11.6.

\subsection{Obtaining equations of the element faces}

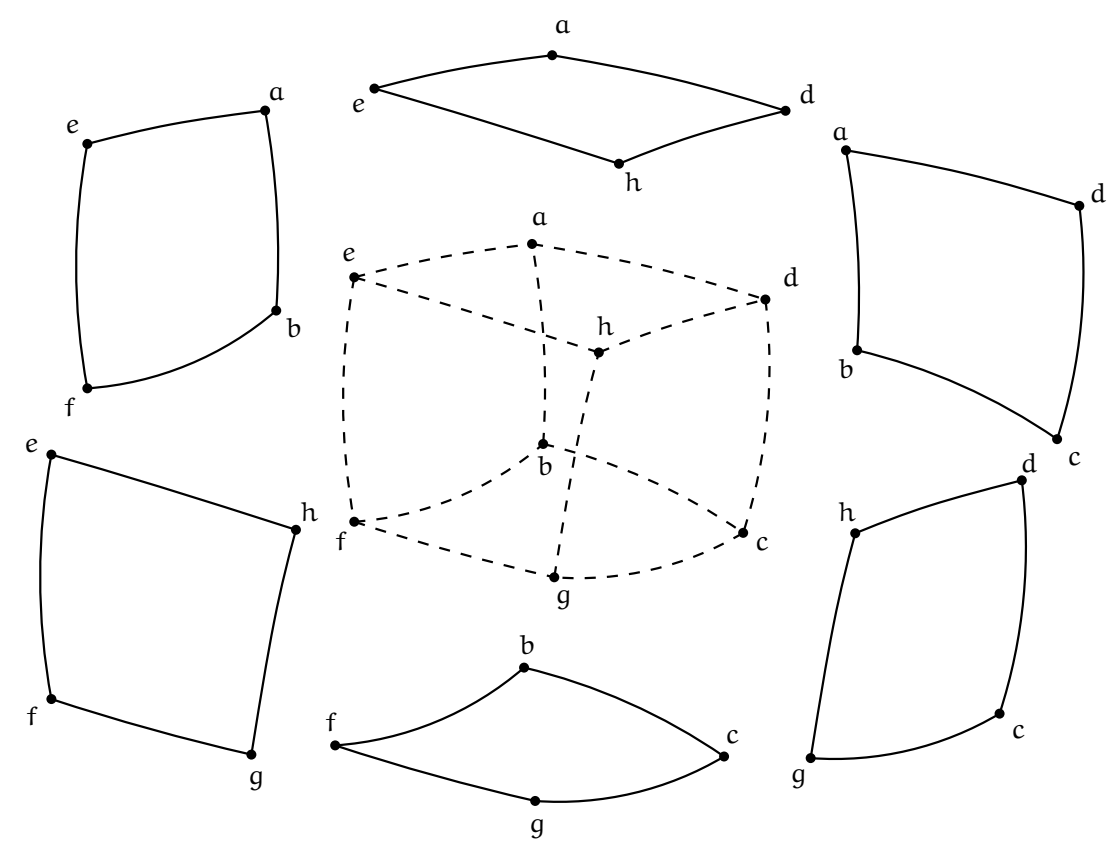

FIG. 8: A representation of hexahedral element $E \equiv a b c d e f g h$ with its six faces $a e h d, a b f e, e h g f, b c g f, c d h g$ and $a d c b$. The coordinate information of the four vertices of each of the faces is used to obtain its equation.

As the procedure invoked in obtaining the equations of the element faces applies to both $E^{f}$ and $E^{p}$, we use the notation $E$ for hexahedral element without regard to whether it is a flow element $E^{f}$ or poromechanics element $E^{p}$. Let $\mathcal{S}(\mathbf{x})=0, \mathbf{x} \equiv(x, y, z) \in e$ be the equation of face $e$ of element $E$ with its vertices $\mathbf{v}_{i} \equiv\left(x_{i}, y_{i}, z_{i}\right)$, $i=1,2,3,4$. A representation of $E$ with its faces is provided in Figure 8. Define $\mathcal{S}(\mathbf{x})$ by a trilinear as

$$
\mathcal{S}(\mathbf{x})=\left\{\begin{array}{llllllll}
x y z & x y & y z & x z & x & y & z & 1
\end{array}\right\} \mathbf{c}_{8 \times 1}
$$

where $\mathbf{c}_{8 \times 1}$ is the vector of coefficients to be determined. Since $\mathcal{S}(\mathbf{x})=0$ is satisfied at each of the four vertices defining the face, we get the system of equations for $\mathbf{c}$

$$
\overbrace{\left[\begin{array}{llllllll}
x_{1} y_{1} z_{1} & x_{1} y_{1} & y_{1} z_{1} & x_{1} z_{1} & x_{1} & y_{1} & z_{1} & 1 \\
x_{2} y_{2} z_{2} & x_{2} y_{2} & y_{2} z_{2} & x_{2} z_{2} & x_{2} & y_{2} & z_{2} & 1 \\
x_{3} y_{3} z_{3} & x_{3} y_{3} & y_{3} z_{3} & x_{3} z_{3} & x_{3} & y_{3} & z_{3} & 1 \\
x_{4} y_{4} z_{4} & x_{4} y_{4} & y_{4} z_{4} & x_{4} z_{4} & x_{4} & y_{4} & z_{4} & 1
\end{array}\right]}^{\mathbf{M}_{4 \times 8}} \mathbf{c}_{8 \times 1}=\left\{\begin{array}{l}
0 \\
0 \\
0 \\
0
\end{array}\right\}_{4 \times 1}
$$

It is clear that $\mathbf{c}$ belongs to the nullspace of $\mathbf{M}$. With that in mind, we get the SVD of $\mathbf{M}$ as

$$
\mathbf{M}_{4 \times 8}=\mathbf{U}_{4 \times 4} \boldsymbol{\sigma}_{4 \times 8} \mathbf{V}_{8 \times 8}^{T}
$$


where $\boldsymbol{\sigma}=\operatorname{diag}\left(\sigma_{1}, . ., \sigma_{r}\right)$ is diagonal matrix of singular values of $\mathbf{M}$ and the columns of $\mathbf{U}$ and $\mathbf{V}$ are left and right singular vectors of $\mathbf{M}$ respectively. Since the nullspace of $\mathbf{M}$ is spanned by right singular vectors corresponding to the vanishing singular values of $\mathbf{M}$, we express $\mathbf{c}$ as

$$
\mathbf{c}_{8 \times 1}=[\mathbf{V}[:, r+1] \quad . \quad \mathbf{V}[:, 8]]_{8 \times(8-r)} \mathbf{K}_{(8-r) \times 1}
$$

where $\boldsymbol{\kappa}$ is the vector of coefficients and $r$ is rank of $\mathbf{M}$. The objective now is to determine $\kappa$. First, using (55), we obtain an expression for the gradient $\nabla \mathcal{S}(\mathbf{x})$ of $\mathcal{S}(\mathbf{x})$ as

$$
\nabla \mathcal{S}(\mathbf{x})=\overbrace{\left[\begin{array}{llllllll}
y z & y & 0 & z & 1 & 0 & 0 & 0 \\
x z & x & z & 0 & 0 & 1 & 0 & 0 \\
x y & 0 & y & x & 0 & 0 & 1 & 0
\end{array}\right]}^{\mathbf{H}(x, y, z)_{3 \times 8}}[\mathbf{V}[:, r+1] \quad . \quad \cdot \mathbf{V}[:, 8]]_{8 \times(8-r)} \mathbf{K}_{(8-r) \times 1}
$$

Let $\hat{\mathcal{S}}(\hat{\mathbf{x}})$ be corresponding definition on face $\hat{e}$ of reference element $\hat{E}$ of $\mathcal{S}(\mathbf{x})$ on face $e$ of actual element $E$. Then, from (15) and (16),

$$
\nabla \mathcal{S}(\mathbf{x})=\left(D F_{E}\right)^{-T}(\hat{\mathbf{x}}) \hat{\nabla} \hat{\mathcal{S}}(\hat{e})
$$

where $\hat{\nabla} \hat{\mathcal{S}}(\hat{e})$ can be either $\left[\begin{array}{lll}1 & 0 & 0\end{array}\right]^{T},\left[\begin{array}{lll}0 & 1 & 0\end{array}\right]^{T}$ or $\left[\begin{array}{lll}0 & 0 & 1\end{array}\right]^{T}$ depending on whether $\hat{e}$ is normal to $\hat{x}, \hat{y}$ or $\hat{z}$ axis. Equating (58) and (59) for all four vertices of $e \in E$, we get the following system of equations for $\boldsymbol{\kappa}_{(8-r) \times 1}$

$$
\left[\begin{array}{l}
\mathbf{H}\left(x_{1}, y_{1}, z_{1}\right) \\
\mathbf{H}\left(x_{2}, y_{2}, z_{2}\right) \\
\mathbf{H}\left(x_{3}, y_{3}, z_{3}\right) \\
\mathbf{H}\left(x_{4}, y_{4}, z_{4}\right)
\end{array}\right]_{12 \times 8}[\mathbf{V}[:, r+1] \quad . \quad \cdot \mathbf{V}[:, 8]]_{8 \times(8-r)} \mathbf{K}_{(8-r) \times 1}=\mathbf{B}_{12 \times 1}
$$

where $\mathbf{B}$ is obtained as

$$
\mathbf{B}[(i-1) * 3+1 \rightarrow i * 3,1]=\left(D F_{E}\right)^{-T}\left(\hat{\mathbf{v}}_{\mathbf{i}}\right) \hat{\nabla} \hat{\mathcal{S}}(\hat{e})
$$

where $\hat{\mathbf{v}}_{\mathbf{i}}, i=1,2,3,4$ on $\hat{e} \in \hat{E}$ is the corresponding definition of $\mathbf{v}_{i}, i=1,2,3,4$ on $e \in E$. The solution $\kappa$ of (60) is substituted into (57) to obtain $\mathbf{c}$, which is then substituted into (55) to obtain the polynomial expression of $\mathcal{S}(\mathbf{x})$.

\subsection{Obtaining points on the periphery of intersection polyhedron using surface-surface intersections}

Algorithm 3 obtains points on the periphery of $\mathcal{E}$ by tracing the intersections of the faces of $E^{f}$ and $E^{p}$. The starting points for the curve traces are the set of vertices of $E^{f}$ inside $E^{p}$.

To understand the construct of the algorithm, we refer to a case depicted in 9. Red arrows represent the set $\mathcal{Q}^{f f}$ i.e. traces of the intersections of the faces of $E^{f}$. Blue arrows represent the set $\mathcal{Q}^{f p}$ i.e. traces of the intersections of the faces of $E^{f}$ with the faces of $E^{p}$. Green arrows represent the set $\mathcal{Q}^{p p}$ i.e. traces of the intersections of the faces of $E^{p}$. The nested loop structure in Algorithm 3 traces out elements of the set $\mathcal{Q}_{f f}, \mathcal{Q}_{f p}$ and $\mathcal{Q}_{p p}$ sequentially. Representing $\mathcal{S}_{x-}^{f}, \mathcal{S}_{x+}^{f}, \mathcal{S}_{y-}^{f}, \mathcal{S}_{y+}^{f}, \mathcal{S}_{z-}^{f}$ and $\mathcal{S}_{z+}^{f}$ as the faces of the flow element normal to the $x-, x+, y-, y+, z-$ and $z+$ axes respectively and $\mathcal{S}_{x-}^{p}, \mathcal{S}_{x+}^{p}, \mathcal{S}_{y-}^{p}, \mathcal{S}_{y+}^{p}, \mathcal{S}_{z-}^{p}$ and $\mathcal{S}_{z+}^{p}$ as the faces of the poromechanics element normal to the $x-, x+, y-, y+, z-$ and $z+$ axes respectively, the curve traces are

$$
\begin{aligned}
& A D \equiv \mathcal{S}_{x-}^{f} \cap \mathcal{S}_{z+}^{f} \rightarrow D H \equiv \mathcal{S}_{z+}^{f} \cap \mathcal{S}_{y+}^{p} \\
& E F \equiv \mathcal{S}_{x+}^{f} \cap \mathcal{S}_{y-}^{f} \rightarrow F B \equiv \mathcal{S}_{y-}^{f} \cap \mathcal{S}_{z-}^{p} \\
& A B \equiv \mathcal{S}_{y-}^{f} \cap \mathcal{S}_{x-}^{f} \rightarrow B C \equiv \mathcal{S}_{x-}^{f} \cap \mathcal{S}_{z-}^{p} \rightarrow C G \equiv \mathcal{S}_{z-}^{p} \cap \mathcal{S}_{y+}^{p} \\
& E A \equiv \mathcal{S}_{y-}^{f} \cap \mathcal{S}_{z-}^{f} \\
& E H \equiv \mathcal{S}_{z+}^{f} \cap \mathcal{S}_{x+}^{f} \rightarrow H G \equiv \mathcal{S}_{x+}^{f} \cap \mathcal{S}_{y+}^{p} \rightarrow G C \equiv \mathcal{S}_{y+}^{p} \cap \mathcal{S}_{z-}^{p}
\end{aligned}
$$

(Sequence 1, Starting point $A$ on $\mathcal{S}_{x-}^{f}$ )

(Sequence 2, Starting point $E$ on $\mathcal{S}_{x+}^{f}$ )

(Sequence 3, Starting point $A$ on $\mathcal{S}_{y-}^{f}$ )

(Sequence 4, Starting point $E$ on $\mathcal{S}_{z-}^{f}$ )

(Sequence 5 , Starting point $E$ on $\mathcal{S}_{z+}^{f}$ ) 

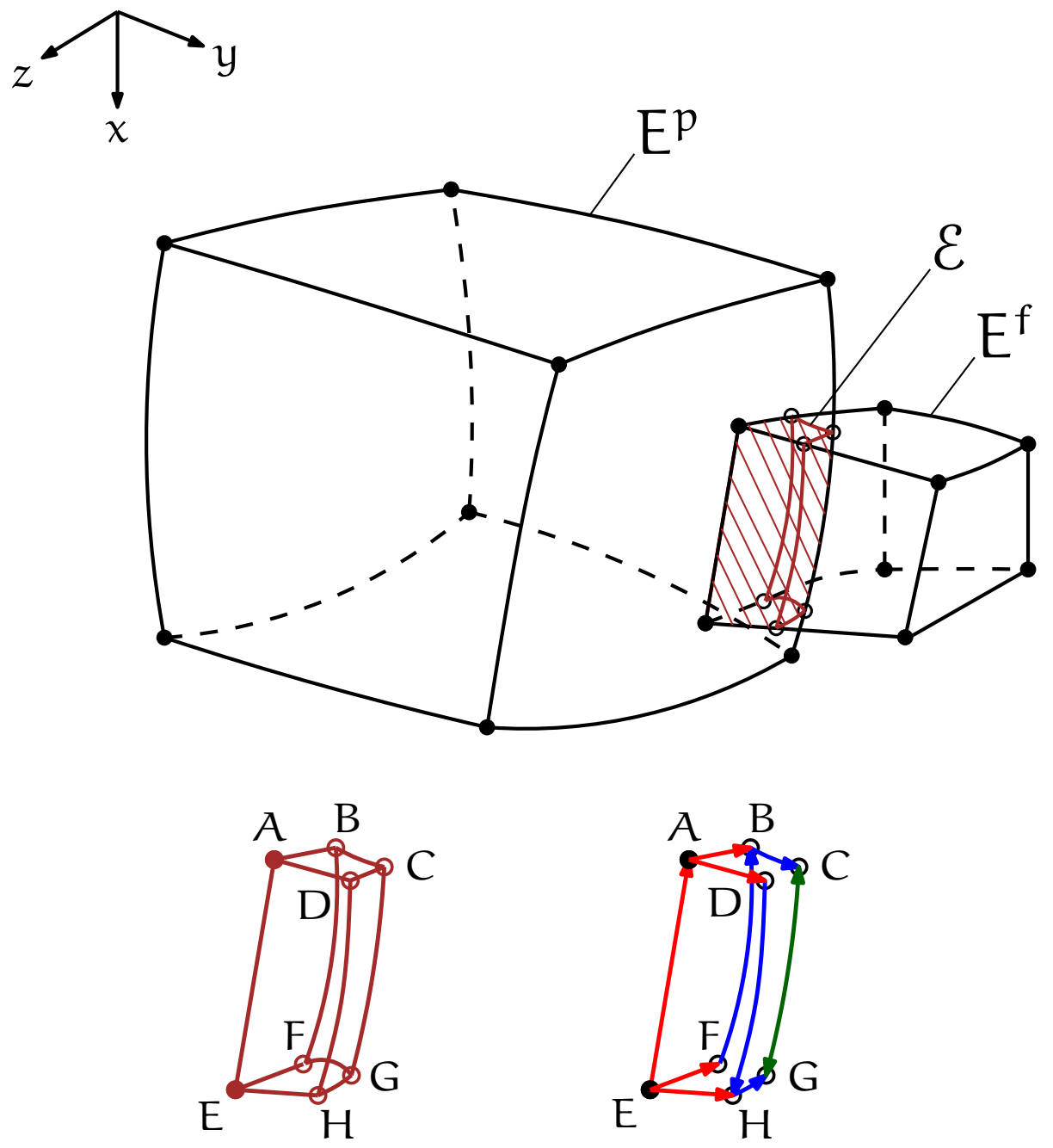

FIG. 9: Solid circles representing points $A$ and $E$ are the vertices of $E^{f}$ inside $E^{p}$. Hollow circles representing points $B, C$, $D, E, F, G$ and $H$ are the end points of the curve traces obtained using the surface-surface intersections algorithm. The arrows represent the direction of the curve traces. It is important to note that Figure 9 is only a depiction of $\mathcal{E}$ and that there is no restriction whatsoever that it be 8-noded.

\subsection{Curve tracing : the function $\operatorname{TRACE}\left(\mathbf{q}, \mathcal{S}_{1}, \mathcal{S}_{2}\right)$}

The curve tracing is based on the predictor-corrector approach of [36]. The predictor gives a second order Taylor approximant to the trace of the intersection curve and the corrector refines the approximant to a point on the intersection curve using the Newton method. With reference to Figure 10, a predictor to the trace of the intersection of surfaces $\mathcal{S}_{1}$ and $\mathcal{S}_{2}$ is stored in $\mathbf{q}_{p}$ and the corrector to $\mathbf{q}_{p}$ is stored in $\mathbf{q}_{c}$.

\subsection{Obtaining $\mathrm{q}_{p}$}

The intersection curve $\mathbf{r}$ of $\mathcal{S}_{1}$ and $\mathcal{S}_{2}$ with initial point $\mathbf{q}$ is expressed as

$$
\mathbf{r}(s)=\mathbf{q}+s \mathbf{r}^{\prime}(\mathbf{q})+\frac{s^{2}}{2 !} \mathbf{r}^{\prime \prime}(\mathbf{q})+\mathbf{e}(s)=\mathbf{q}_{p}(s)+\mathbf{e}(s)
$$



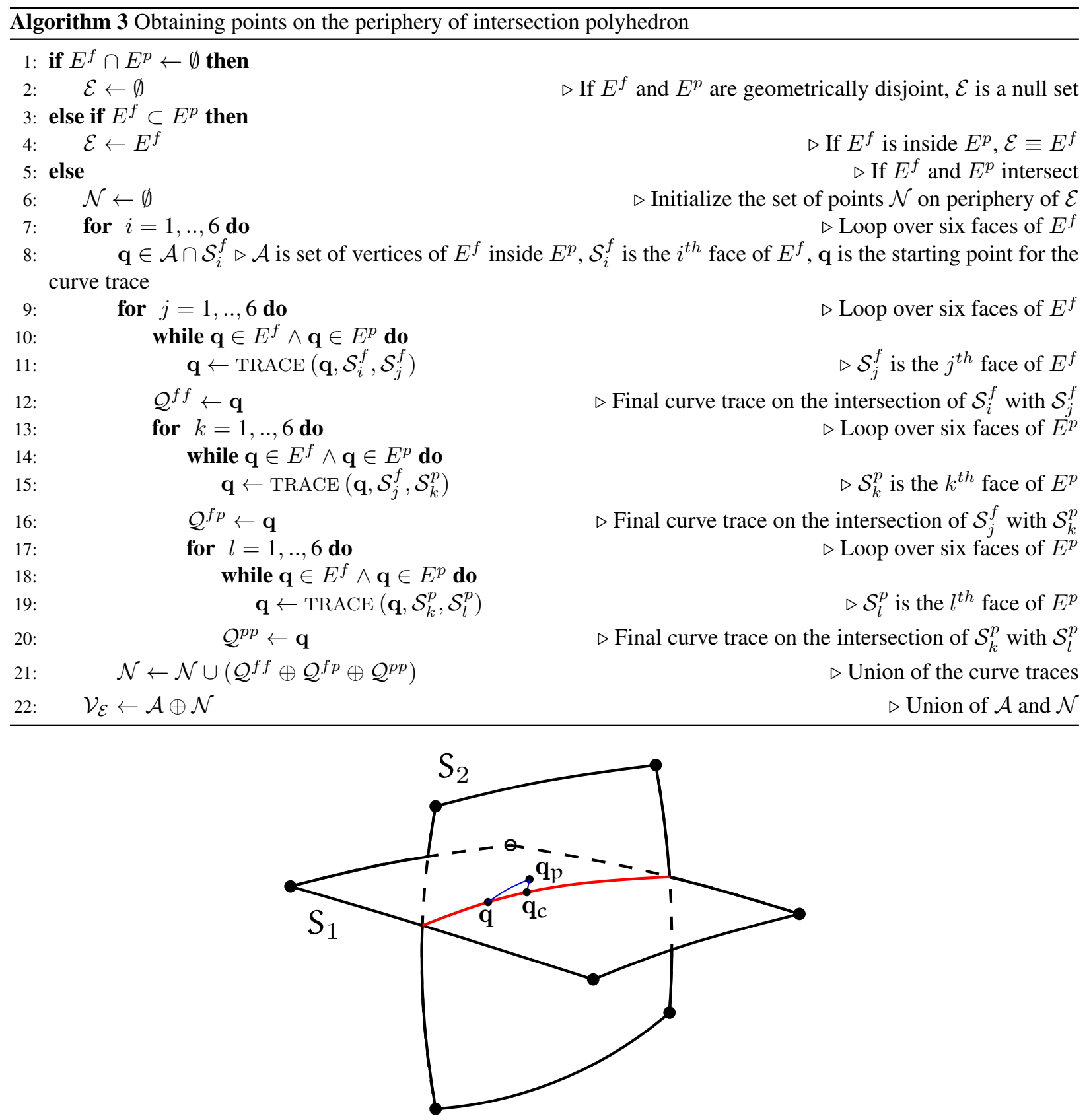

FIG. 10: $\mathcal{S}_{1} \cap \mathcal{S}_{2}$ represented by red solid line. $\mathbf{q}_{p}$ is the predictor to the trace of $\mathcal{S}_{1} \cap \mathcal{S}_{2}$. $\mathbf{q}_{c}$ is the corrector to $\mathbf{q}_{p}$.

where $s$ is an arc length parameter, $\mathbf{r}^{\prime}(\mathbf{q})$ is unit tangent to curve at $\mathbf{q}, \mathbf{r}^{\prime \prime}(\mathbf{q})$ is curvature at $\mathbf{q}$ and $\mathbf{e}(s)=O\left(s^{3}\right)$ is the error. We assume $s=0.1$, a value small enough to make $\mathbf{q}_{p}(s)$ an accurate estimate of $\mathbf{r}(s)$ i.e. $|\mathbf{e}(s)|<<|\mathbf{p}(s)|$. As long as $\mathbf{q}$ is not singular on $\mathcal{S}_{1}$ or on $\mathcal{S}_{2}$, the surface gradients $\nabla \mathcal{S}_{1}(\mathbf{q})$ and $\mathcal{S}_{2}(\mathbf{q})$ are linearly independent (see [36]) and the unit tangent vector $\mathbf{r}^{\prime}(\mathbf{q})$ is obtained as

$$
\mathbf{r}^{\prime}(\mathbf{q})=\frac{\nabla \mathcal{S}_{1}(\mathbf{q}) \times \nabla \mathcal{S}_{2}(\mathbf{q})}{\left\|\nabla \mathcal{S}_{1}(\mathbf{q}) \times \nabla \mathcal{S}_{2}(\mathbf{q})\right\|}
$$


It follows that $\mathbf{r}^{\prime}(\mathbf{q})$ is perpendicular to both the surface gradients such that

$$
\nabla \mathcal{S}_{1}(\mathbf{q}) \cdot \mathbf{r}^{\prime}(\mathbf{q})=\nabla \mathcal{S}_{2}(\mathbf{q}) \cdot \mathbf{r}^{\prime}(\mathbf{q})=0
$$

implying the vectors $\nabla \mathcal{S}_{1}(\mathbf{q}), \nabla \mathcal{S}_{2}(\mathbf{q})$ and $\mathbf{r}^{\prime}(\mathbf{q})$ are linearly independent. We express $\mathbf{r}^{\prime \prime}(\mathbf{q})$ as a linear combination of $\nabla \mathcal{S}_{1}(\mathbf{q}), \nabla \mathcal{S}_{2}(\mathbf{q})$ and $\mathbf{r}^{\prime}(\mathbf{q})$ as

$$
\mathbf{r}^{\prime \prime}(\mathbf{q})=\alpha \mathbf{r}^{\prime}(\mathbf{q})+\beta \nabla \mathcal{S}_{1}(\mathbf{q})+\gamma \nabla \mathcal{S}_{2}(\mathbf{q})
$$

The points on $\mathbf{r}(s)$ are solutions of $\mathcal{S}_{1}(\mathbf{r}(s))=\mathcal{S}_{1}(\mathbf{r}(s))=0$. The Taylor expansion of $\mathcal{S}_{j}(\mathbf{r}(s)), j=1,2$ with $\mathbf{q} \equiv \mathbf{r}^{\prime}(0)$ is

$$
\mathcal{S}_{j}(\mathbf{r}(s))=\mathcal{S}_{j}(\mathbf{q})+s \nabla \mathcal{S}_{j}(\mathbf{q}) \cdot \mathbf{r}^{\prime}(\mathbf{q})+\frac{s^{2}}{2 !}\left(\nabla \mathcal{S}_{j}(\mathbf{q}) \cdot \mathbf{r}^{\prime \prime}(\mathbf{q})+\mathbf{r}^{\prime}(\mathbf{q}) \cdot H_{\mathcal{S}_{j}}(\mathbf{q}) \cdot \mathbf{r}^{\prime}(\mathbf{q})\right)
$$

where $H_{\mathcal{S}_{j}}(\mathbf{q})$ is the Hessian of the surface $\mathcal{S}_{j}$ evaluated at $\mathbf{q}$ as follows

$$
H_{\mathcal{S}_{j}}(\mathbf{q})=\left[\begin{array}{lll}
\frac{\partial^{2} \mathcal{S}_{j}}{\partial x^{2}} & \frac{\partial^{2} \mathcal{S}_{j}}{\partial x \partial y} & \frac{\partial^{2} \mathcal{S}_{j}}{\partial x \partial z} \\
\frac{\partial^{2} \mathcal{S}_{j}}{\partial y \partial x} & \frac{\partial^{2} \mathcal{S}_{j}}{\partial y^{2}} & \frac{\partial^{2} \mathcal{S}_{j}}{\partial y \partial z} \\
\frac{\partial^{2} \mathcal{S}_{j}}{\partial z \partial x} & \frac{\partial^{2} \mathcal{S}_{j}}{\partial z \partial y} & \frac{\partial^{2} \mathcal{S}_{j}}{\partial z^{2}}
\end{array}\right]_{3 \times 3}
$$

where the diagonal entries of $H_{\mathcal{S}_{j}}(\mathbf{q})$ are zero and the off diagonal entries are

$$
\begin{aligned}
\frac{\partial^{2} \mathcal{S}}{\partial x \partial y} & \equiv \frac{\partial^{2} \mathcal{S}}{\partial y \partial x}=\left\{\begin{array}{llllllll}
z & 1 & 0 & 0 & 0 & 0 & 0 & 0
\end{array}\right\} \mathbf{c} \\
\frac{\partial^{2} \mathcal{S}}{\partial x \partial z} & \equiv \frac{\partial^{2} \mathcal{S}}{\partial z \partial x}=\left\{\begin{array}{llllllll}
y & 0 & 0 & 1 & 0 & 0 & 0 & 0
\end{array}\right\} \mathbf{c} \\
\frac{\partial^{2} \mathcal{S}}{\partial y \partial z} & \equiv \frac{\partial^{2} \mathcal{S}}{\partial z \partial y}=\left\{\begin{array}{llllllll}
x & 0 & 1 & 0 & 0 & 0 & 0 & 0
\end{array}\right\} \mathbf{c}
\end{aligned}
$$

where $x, y$ and $z$ are the coordinates of the point $\mathbf{q}$ and $\mathbf{c}$ is the vector of coefficients corresponding to the definition of $\mathcal{S}_{j}$.

Since $\mathcal{S}_{j}(\mathbf{r}(s)) \equiv 0, j=1,2$, the coefficient of each power of $s$ in $\mathcal{S}_{j}(\mathbf{r}(s))$ must be zero. We already know that the coefficient of $s$ in $\mathcal{S}_{j}(\mathbf{r}(s))$ is zero i.e. $\nabla \mathcal{S}_{j}(\mathbf{q}) \cdot \mathbf{r}^{\prime}(\mathbf{q})=0, j=1,2$. Equating the coefficient of $s^{2}$ in $\mathcal{S}_{j}(\mathbf{r}(s))$ to zero, we get

$$
\begin{aligned}
& \nabla \mathcal{S}_{j}(\mathbf{q}) \cdot \mathbf{r}^{\prime \prime}(\mathbf{q})=-\mathbf{r}^{\prime}(\mathbf{q}) \cdot H_{\mathcal{S}_{j}}(\mathbf{q}) \cdot \mathbf{r}^{\prime}(\mathbf{q}) \\
& \nabla \mathcal{S}_{j}(\mathbf{q}) \cdot\left(\alpha \mathbf{r}^{\prime}(\mathbf{q})+\beta \nabla \mathcal{S}_{1}(\mathbf{q})+\gamma \nabla \mathcal{S}_{2}(\mathbf{q})\right)=-\mathbf{r}^{\prime}(\mathbf{q}) \cdot H_{\mathcal{S}_{j}}(\mathbf{q}) \cdot \mathbf{r}^{\prime}(\mathbf{q})
\end{aligned}
$$

and noting again that $\nabla \mathcal{S}_{j}(\mathbf{q}) \cdot \mathbf{r}^{\prime}(\mathbf{q})=0, j=1,2$, we get the following system of equations for $\beta$ and $\gamma$

$$
\left[\begin{array}{cc}
\nabla \mathcal{S}_{1}(\mathbf{q}) \cdot \nabla \mathcal{S}_{1}(\mathbf{q}) & \nabla \mathcal{S}_{1}(\mathbf{q}) \cdot \nabla \mathcal{S}_{2}(\mathbf{q}) \\
\nabla \mathcal{S}_{2}(\mathbf{q}) \cdot \nabla \mathcal{S}_{1}(\mathbf{q}) & \nabla \mathcal{S}_{2}(\mathbf{q}) \cdot \nabla \mathcal{S}_{2}(\mathbf{q})
\end{array}\right]\left\{\begin{array}{l}
\beta \\
\gamma
\end{array}\right\}=-\left\{\begin{array}{l}
\mathbf{r}^{\prime}(\mathbf{q}) \cdot H_{\mathcal{S}_{1}}(\mathbf{q}) \cdot \mathbf{r}^{\prime}(\mathbf{q}) \\
\mathbf{r}^{\prime}(\mathbf{q}) \cdot H_{\mathcal{S}_{2}}(\mathbf{q}) \cdot \mathbf{r}^{\prime}(\mathbf{q})
\end{array}\right\}
$$

Solution of (63) and the choice $\alpha=0$ leads to a unique vector $\mathbf{r}^{\prime \prime}(\mathbf{q})$ in (62). The second order interpolant $\mathbf{q}_{p}(s)$ is finally obtained as

$$
\mathbf{q}_{p}(s)=\mathbf{q}+0.1 \frac{\nabla \mathcal{S}_{1}(\mathbf{q}) \times \nabla \mathcal{S}_{2}(\mathbf{q})}{\left\|\nabla \mathcal{S}_{1}(\mathbf{q}) \times \nabla \mathcal{S}_{2}(\mathbf{q})\right\|}+\frac{0.01}{2 !}\left(\beta \nabla \mathcal{S}_{1}(\mathbf{q})+\gamma \nabla \mathcal{S}_{2}(\mathbf{q})\right)
$$




\subsection{Obtaining $\mathrm{q}_{c}$}

Given the quadratic interpolant to the curve at $\mathbf{q}_{p}$ in (64), we refine its estimate to a point on the curve by generating a sequence of points $\mathbf{q}_{1}, \mathbf{q}_{2}, \cdots \rightarrow \mathbf{q}_{c}$ with $\mathbf{q}_{0}=\mathbf{q}_{p}$. The Newton method for the solution of $\mathcal{S}_{j}(\mathbf{r}(s))=0, j=1,2$ at $\mathbf{r}(s)=\mathbf{q}_{k}$ is

$$
\nabla \mathcal{S}_{j}\left(\mathbf{q}_{k}\right) \cdot\left(\mathbf{q}_{k+1}-\mathbf{q}_{k}\right)=-\mathcal{S}_{j}\left(\mathbf{q}_{k}\right)
$$

where $k$ is the iteration count. Expressing $\boldsymbol{\Delta}_{k} \equiv \mathbf{q}_{k+1}-\mathbf{q}_{k}$ as a linear combination of $\mathbf{r}^{\prime}\left(\mathbf{q}_{k}\right), \nabla \mathcal{S}_{1}\left(\mathbf{q}_{k}\right)$ and $\nabla \mathcal{S}_{2}\left(\mathbf{q}_{k}\right)$ as

$$
\boldsymbol{\Delta}_{k}=\varsigma_{k} \mathbf{r}^{\prime}\left(\mathbf{q}_{k}\right)+\vartheta_{k} \nabla \mathcal{S}_{1}\left(\mathbf{q}_{k}\right)+\varphi_{k} \nabla \mathcal{S}_{2}\left(\mathbf{q}_{k}\right)
$$

and substituting in (65) results in

$$
\nabla \mathcal{S}_{j}\left(\mathbf{q}_{k}\right) \cdot\left(\varsigma_{k} \mathbf{r}^{\prime}\left(\mathbf{q}_{k}\right)+\vartheta_{k} \nabla \mathcal{S}_{1}\left(\mathbf{q}_{k}\right)+\varphi_{k} \nabla \mathcal{S}_{2}\left(\mathbf{q}_{k}\right)\right)=-\mathcal{S}_{j}\left(\mathbf{q}_{k}\right)
$$

The choice $\varsigma_{k}=0$ leads to the following system of equations for $\vartheta_{k}$ and $\varphi_{k}$

$$
\left[\begin{array}{ll}
\nabla \mathcal{S}_{1}\left(\mathbf{q}_{k}\right) \cdot \nabla \mathcal{S}_{1}\left(\mathbf{q}_{k}\right) & \nabla \mathcal{S}_{1}\left(\mathbf{q}_{k}\right) \cdot \nabla \mathcal{S}_{2}\left(\mathbf{q}_{k}\right) \\
\nabla \mathcal{S}_{2}\left(\mathbf{q}_{k}\right) \cdot \nabla \mathcal{S}_{1}\left(\mathbf{q}_{k}\right) & \nabla \mathcal{S}_{2}\left(\mathbf{q}_{k}\right) \cdot \nabla \mathcal{S}_{2}\left(\mathbf{q}_{k}\right)
\end{array}\right]\left\{\begin{array}{c}
\vartheta_{k} \\
\varphi_{k}
\end{array}\right\}=-\left\{\begin{array}{l}
\mathcal{S}_{1}\left(\mathbf{q}_{k}\right) \\
\mathcal{S}_{2}\left(\mathbf{q}_{k}\right)
\end{array}\right\}
$$

Solution of (67) along with (66) is used to obtain $\Delta_{k}$. The Newton method (65) is iterated until a convergence criterion is met as shown in Algorithm 4.

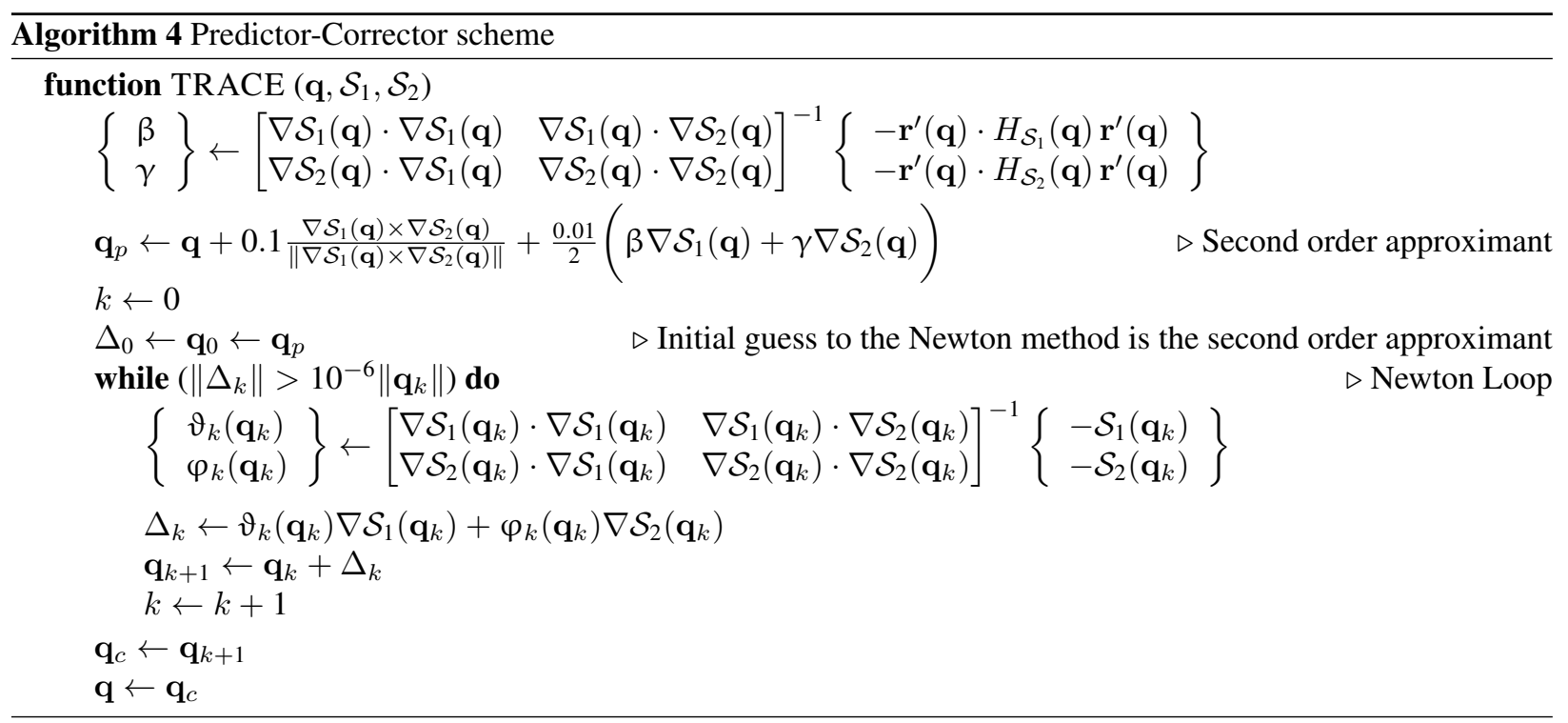

\subsection{Obtaining volume of intersection polyhedron using Delaunay triangulation}

We use a library code TetGen (see [37]) for this purpose. The library code takes as input the coordinates of the set of points on the periphery of intersection polyhedron and decomposes the polyhedron into multiple 3-simplices or tetrahedra. This process is refered to as Delaunay triangulation. Let $\mathcal{D}_{\mathcal{E}}$ be the Delaunay triangulation of $\mathcal{E}$ consisting of tetrahedra $T$ such that $\mathcal{E}=\bigcup_{T \in \mathcal{D}_{\mathcal{E}}} T$. Denoting $\mathbf{v}_{0}, \mathbf{v}_{1}, \mathbf{v}_{2}$ and $\mathbf{v}_{3}$ as position vectors of the vertices of $T$ and $\left(\mathbf{v}_{1}-\mathbf{v}_{0}\right),\left(\mathbf{v}_{2}-\mathbf{v}_{0}\right)$ and $\left(\mathbf{v}_{3}-\mathbf{v}_{0}\right)$ as columns of the $3 \times 3$ matrix $\mathcal{X}^{T}$, we get

$$
\operatorname{Meas}(\mathcal{E})=\sum_{T \in \mathcal{D}} \operatorname{Meas}(T)=\sum_{T \in \mathcal{D}} \frac{1}{3 !} \operatorname{det}\left(\mathcal{X}^{T}\right)
$$




\section{SUMMARY OF STEPS INVOLVED IN CONSTRUCTION OF MAPPING OPERATORS}

The process of obtaining the mapping operators is summarized here

$\checkmark$ Identify each pair of overlapping flow and poromechanics elements.

$\checkmark$ For each such pair, obtain the equations of the faces of the flow and poromechanics element.

$\checkmark$ Obtain the points on the periphery of the intersection polyhedron of the aforementioned pair using surfacesurface intersections.

$\checkmark$ With points on the periphery of the intersection polyhedron as input, obtain the volume of the intersection polyhedron using Delaunay triangulation.

$\checkmark$ Use the obtained volume to construct the local mapping operators.

\section{VERIFICATION: MANDEL'S PROBLEM SOLUTION FOR CONSOLIDATION IN HOMOGENEOUS POROUS MEDIUM}

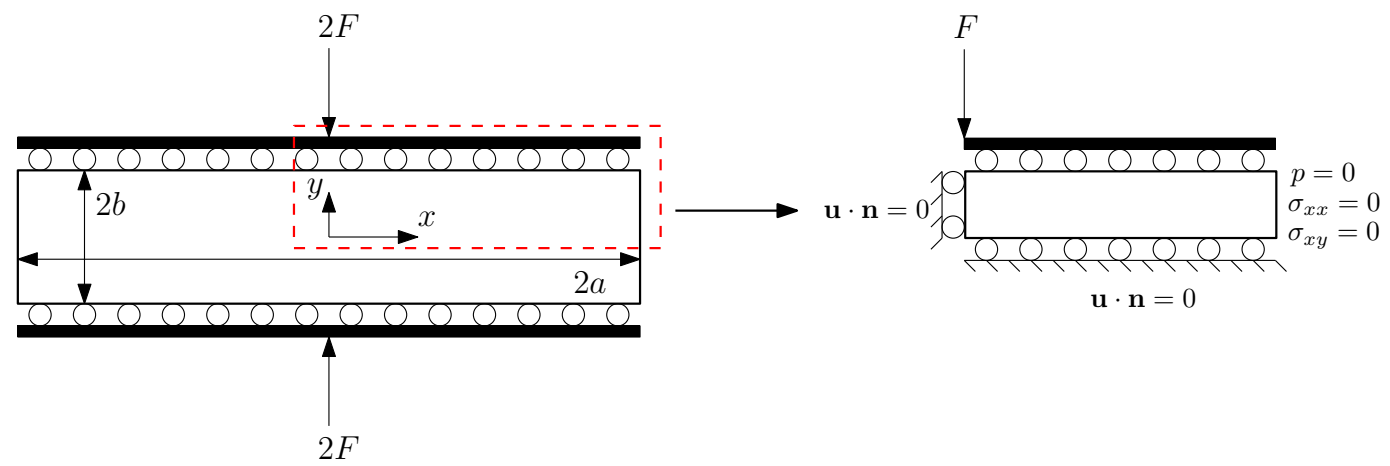

FIG. 11: Circles indicate rollers and solid black boxes indicate rigid frictionless plates. The biaxial symmetry of the problem allows us to replicate the problem by only modeling a quarter of the domain as indicated by the red dotted line.

Solve flow on fine mesh

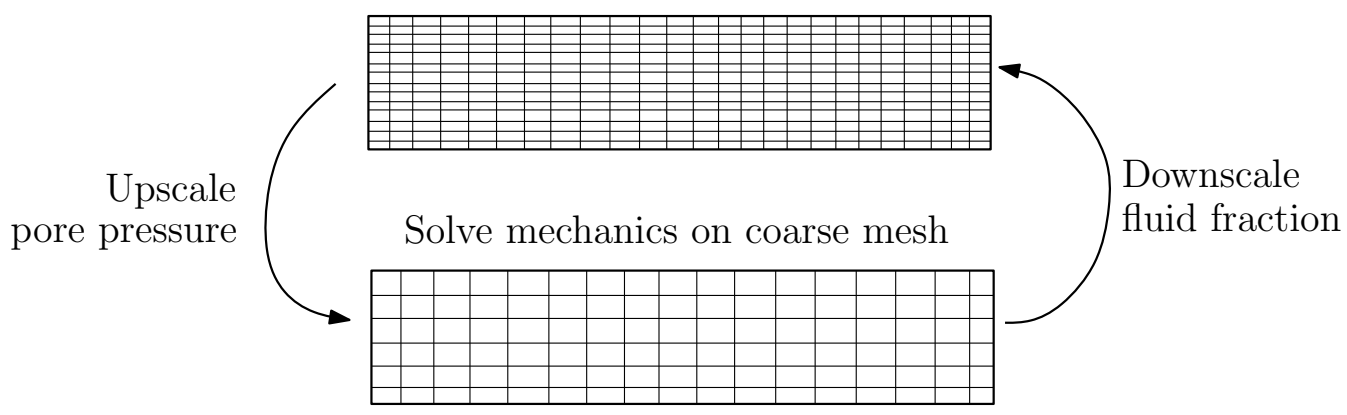

FIG. 12: Solution methodology

[38] solved the Biot equations for the case of a rectangular porous specimen with incompressible pore fluid in plane strain conditions sandwiched between frictionless plates and subjected to a point load with free drainage on the lateral sides. He arrived at a non-monotonic pore pressure solution at the center of a specimen i.e. an increase in pore pressure from $t=0^{+}$until a certain time at which the pressure reached a maximum followed by a monotonic decrease. [39] arrived at a similar non-monotonic pore pressure response at the center of water saturated sphere of soil 


\begin{tabular}{c|c|c}
\hline Parameter & Quantity & Value \\
\hline$a$ & x dimension & $100 \mathrm{~m}$ \\
\hline$b$ & y dimension & $10 \mathrm{~m}$ \\
\hline$E$ & Young's Modulus & $5.94 \times 10^{9} \mathrm{~Pa}$ \\
\hline$v$ & Poisson's ratio & 0.2 \\
\hline$v_{u}$ & Undrained Poisson's ratio & 0.3846 \\
\hline$\alpha$ & Biot parameter & 0.8 \\
\hline$k$ & Permeability & $100 \mathrm{md}$ \\
\hline$B$ & Skempton coefficient & 0.8333 \\
\hline$c$ & Fluid compressibility & $3.03 \times 10^{-10} \mathrm{~Pa}^{-1}$ \\
\hline$\phi_{0}$ & Initial porosity & 0.2 \\
\hline$\mu$ & Fluid viscosity & $1.0 \mathrm{cp}$ \\
\hline$\rho_{0}$ & Reference fluid density & $62.4 \mathrm{lbm} / \mathrm{ft}^{3}$ \\
\hline$F$ & Point load intensity & $5.94 \times 10^{8} \mathrm{~N} / \mathrm{m}$ \\
\hline
\end{tabular}

TABLE 1: Parameters for Mandel's problem

\begin{tabular}{c|c|c|c}
\hline$T_{h}^{f}$ & $T_{H}^{p}$ & $\left\|\frac{1}{M}\left(p-p_{H}\right)\right\|_{L^{\infty}\left(L^{2}\right)}$ & Rate \\
\hline $15 \times 15$ & $6 \times 6$ & $0.459 \times 10^{-1}$ & - \\
\hline $20 \times 20$ & $8 \times 8$ & $0.339 \times 10^{-1}$ & 1.053 \\
\hline $25 \times 25$ & $10 \times 10$ & $0.265 \times 10^{-1}$ & 1.104 \\
\hline $30 \times 30$ & $12 \times 12$ & $0.213 \times 10^{-1}$ & 1.198 \\
\hline
\end{tabular}

TABLE 2: Order of convergence of upscaled pore pressure solution using the two grid fixed stress split iterative scheme for the Mandel's problem.

subjected to uniform hydrostatic pressure with free drainage at the surface. As a result, the phenomenon is commonly refered to as the 'Mandel-Cryer effect'. He observed that a monotonic pore pressure response is obtained only in the singular case when the shear modulus of the skeleton is zero. He also used the three-dimensional Terzaghi theory to arrive at the pore pressure response for the same configuration and observed that in that case, the water pressure drops steadily. He also showed that when the Poisson's ratio of the skeleton is 0.5 , the Biot model predicts zero volume change whereas the Terzaghi theory predicts non-zero volume changes. The Mandel-Cryer effect was later experimentally verified by [40] and [41]. The reproducibility of this effect was the primary response why the Biot theory gained popularity in comparison to the Terzaghi theory. Later, [42] obtained an analytical solution for the Mandel's problem but with the pore fluid compressibility factored in. This solution commonly serves as a benchmark for validation of coupled flow and poroelasticity codes. In our two grid implementation, the flow and mechanics domains, although identical, have different finite element discretizations, with mechanics being resolved on a coarser mesh. For the sake of clarity, we write the governing equations applicable to the Mandel's problem here as follows

$$
\begin{aligned}
\nabla \cdot\left(\boldsymbol{\sigma}_{0}+\frac{1}{2} \mathbb{D}\left(\nabla \mathbf{u}+\nabla^{T} \mathbf{u}\right)-\alpha\left(p-p_{0}\right) \mathbf{I}\right) & =0 \\
\frac{\partial}{\partial t}\left(\frac{1}{M} p+\nabla \cdot(\alpha \mathbf{u})\right)+\nabla \cdot\left(-\frac{k}{\mu} \nabla p\right) & =0
\end{aligned}
$$

where (68) is the usual linear momentum balance for the solid phase with the small strain assumption in the absence of gravity and (69) is the equation of mass conservation for linearized slightly compressible single phase flow with gravity turned off. As shown in Figure 11, an infinitely long rectangular isotropic specimen is sandwiched between rigid, frictionless plates. The lateral sides are free from normal and shear stress and pore pressure. At $t=0^{+}$, a force 
intensity of $2 F N / m$ is applied to the rigid plates. The initial and boundary conditions are

$$
\begin{array}{r}
\left.\sigma_{x x}\right|_{t=0}=\left.\sigma_{x y}\right|_{t=0}=\left.\sigma_{y y}\right|_{t=0}=0,\left.p\right|_{t=0}=0 \quad \forall x, y \\
\left.\sigma_{x x}\right|_{x= \pm a}=\left.\sigma_{x y}\right|_{x= \pm a}=\left.\sigma_{y x}\right|_{y= \pm b}=0,\left(\int_{-a}^{a} \sigma_{y y} d x\right)_{y= \pm b}=-2 F \quad \forall t \\
\left.p\right|_{x= \pm a}=0,(\mathbf{u} \cdot \mathbf{n})_{y= \pm b}=0 \quad \forall t
\end{array}
$$

where $\mathbf{n}$ is unit outward normal to the boundary. Plane strain condition is applicable i.e. $\epsilon_{z z}=0$. Given the biaxial symmetry of the problem, only a quarter of the domain needs to be modeled. Following the approach of [43], with $U_{y}^{a}(b)$ representing the analytical solution for the $y$ displacement at $y=b$, the boundary conditions are recast as

$$
\begin{array}{rr}
\left.\sigma_{x x}\right|_{x=a}=\left.\sigma_{x y}\right|_{x=a}=\left.\sigma_{y x}\right|_{y=b}=0,(\mathbf{u} \cdot \mathbf{n})_{y=b}=U_{y}^{a}(b) & \forall t \\
(\mathbf{u} \cdot \mathbf{n})_{x=0}=(\mathbf{u} \cdot \mathbf{n})_{y=0}=0,\left.p\right|_{x=a}=0 & \forall t \\
(\mathbf{u} \cdot \mathbf{n})_{x=0}=(\mathbf{u} \cdot \mathbf{n})_{y=0}=(\mathbf{u} \cdot \mathbf{n})_{y=b}=0 & \forall t
\end{array}
$$

We solve the system (69)-(68) using the two grid scheme on rectilinear nonmatching grids as shown in Figure 12 and show its convergence by measuring the upscaled pressure solution error. We employ the parameters given in Table 1 and keep the mesh ratio $r$ fixed. Since the inverse of the Biot modulus $\frac{1}{M}$ is bounded below by a positive constant (as the initial porosity field $\phi_{0}$ is strictly positive), optimality should be achieved when the pressure solution error is measured in the $L^{\infty}\left(L^{2}\right)$ norm (see [44]). The error norm is computed using the midpoint quadrature rule:

$\left\|\frac{1}{M}\left(p-p_{H}\right)\right\|_{L^{\infty}\left(L^{2}\right)} \equiv \max _{0<\tau \leq T}\left(\sum_{E \in T_{H}^{p}}|E|\left(\frac{p\left(\tau, m_{e}\right)-p_{H}\left(m_{e}\right)}{M}\right)^{2}\right)^{\frac{1}{2}}$ where $m_{e}$ is the center of mass of element $E$ and $T$ is the total time. To minimize the effects of the error produced by time discretization, a small time step of $1 \times 10^{-3} \mathrm{sec}$ is chosen. As shown in Table 2, we observe first order convergence for the pore pressure solution with the augmented scheme for $r=2.5$. A fractional value of $r$ ensures the cardinality $|\{\mathcal{E}\}|$ of the set $\{\mathcal{E}\}$ of intersection polyhedra is non-zero i.e. the number of instances of intersecting flow and mechanics elements is not zero. It is important to note that there is no restriction posed on the value of $r$ and we choose a value of 2.5 only for the sake of convenience. We then compare the upscaled pore pressure solution at the cell-center closest to the origin of the quarter domain with the analytical solution for all the above combinations of $T_{h}^{f}, T_{H}^{p}$. The reason for choosing the cell-center closest to the origin of the quarter domain is that the classical non-monotonic pore pressure response, which we intend to replicate in our numerical model, is expected only near the central region of the specimen (see [42]). We also compare the computed $x$-displacement at the free end $x=a$ with the analytical solution. The total simulation time is $50000 \mathrm{sec}$ with a time step of $10 \mathrm{sec}$. According to the analytical solution, at the instant of loading, a uniform pressure rise of $\Delta p\left(x, y, 0^{+}\right)=\frac{F B\left(1+v_{u}\right)}{3 a}$ should be observed. Also, after the initial outward movement of $u_{x}\left(a, y, 0^{+}\right)=\frac{F v_{u}}{2 G}$, the side boundaries will contract toward the center and its final state should be $u_{x}(a, y, \infty)=\frac{F v}{2 G}$. The pore pressure and displacement solutions are non-dimensionalized by multiplying with $\left(\frac{a}{F}\right)$ and $\left(\frac{2 G}{F}\right)$ respectively. As shown in Figures 13 and 14, we observe an excellent match with the expected results for all the above combinations.

\section{LARGE-SCALE PROBLEM}

As shown in Figure 15, the large-scale problem under consideration consists of a reservoir of dimensions $80 \mathrm{ft} \times$ $9400 \mathrm{ft} \times 8800 \mathrm{ft}$ at a depth of approximately $10000 \mathrm{ft}$ with a dip-angle ranging from $1^{\circ}$ to $3^{\circ}$ surrounded by a geomechanical domain of dimensions $13000 \mathrm{ft} \times 9400 \mathrm{ft} \times 8800 \mathrm{ft}$ with an underburden of approximately $3000 \mathrm{ft}$. Sideburdens are neglected for the sake of convenience. The flow mesh $T_{h}^{f} \equiv 20 \times 188 \times 176$ consists of 661760 distorted hexahedral elements of size roughly $4 \mathrm{ft} \times 50 \mathrm{ft} \times 50 \mathrm{ft}$. As explained in Table 3 , the graded geomechanics mesh $T_{H}^{p} \equiv 82 \times 47 \times 47$ consists of 169576 brick elements. At the outset, it is obvious that in the absence of a multiscale framework, the geomechanics mesh with similar resolution for overburden and underburden would need to 

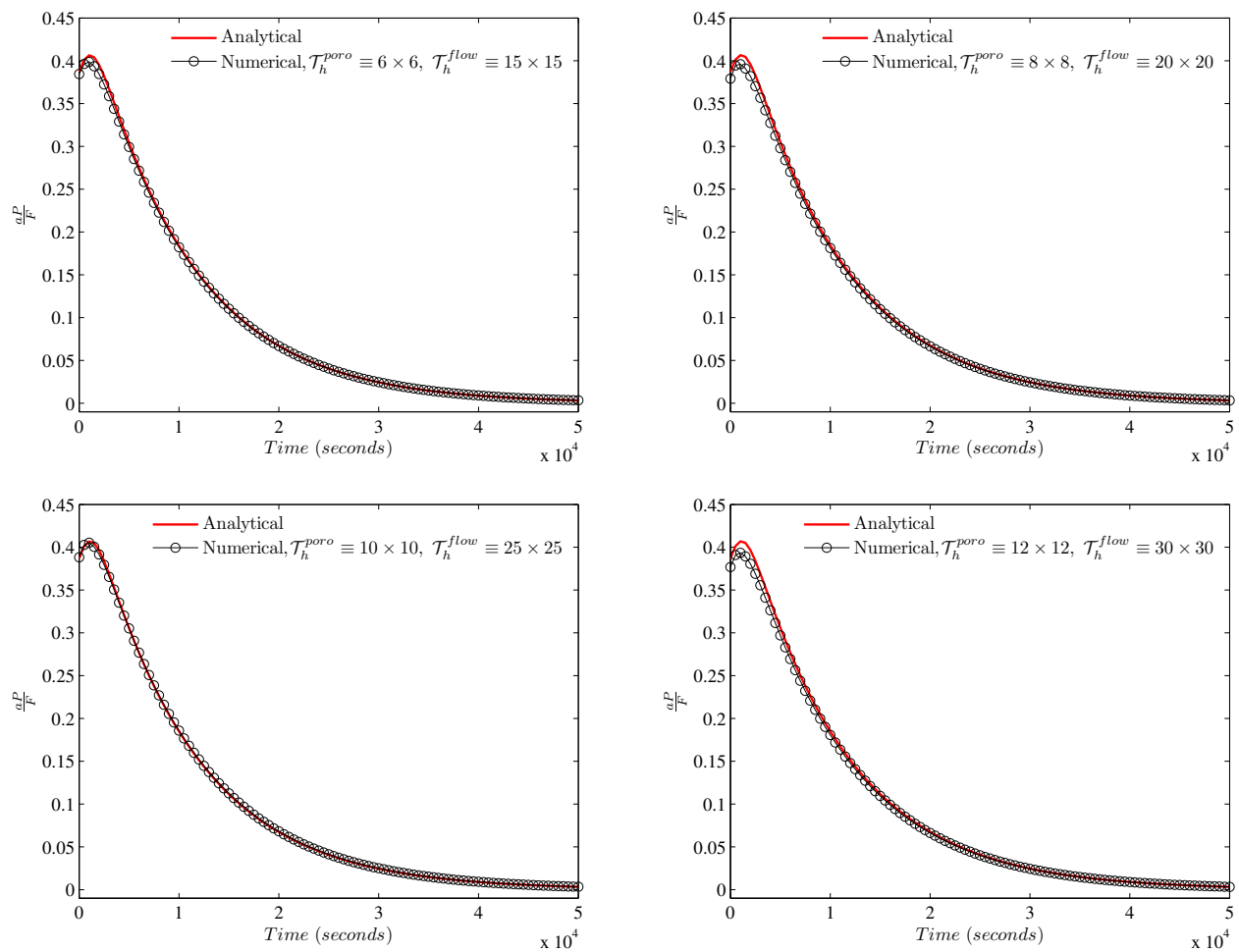

FIG. 13: Non-monotonic pore pressure response at the cell-center closest to the origin for the Mandel's problem with nonmatching grids. $\lim _{t \rightarrow 0^{+}}\left(\frac{a P\left(x_{c}, y_{c}, t\right)}{F}\right)=\frac{B\left(1+v_{u}\right)}{3}=0.3846$ where $x_{c}, y_{c}$ are coordinates of cell-center closest to the origin.

\begin{tabular}{c|c|c|c}
\hline Nature & Depthwise extent & Mesh & Element size \\
\hline Overburden & $0 \mathrm{ft} \rightarrow 9900 \mathrm{ft}$ & $33 \times 47 \times 44$ & $300 \mathrm{ft} \times 200 \mathrm{ft} \times 200 \mathrm{ft}$ \\
\hline Near-reservoir & $9900 \mathrm{ft} \rightarrow 10300 \mathrm{ft}$ & $40 \times 47 \times 44$ & $10 \mathrm{ft} \times 200 \mathrm{ft} \times 200 \mathrm{ft}$ \\
\hline Underburden & $10300 \mathrm{ft} \rightarrow 13000 \mathrm{ft}$ & $9 \times 47 \times 44$ & $300 \mathrm{ft} \times 200 \mathrm{ft} \times 200 \mathrm{ft}$ \\
\hline
\end{tabular}

TABLE 3: Details of the graded geomechanics mesh

conform with the flow grid and would be roughly $T_{H}^{p} \equiv(20+33+7) \times 188 \times 176$ consisting of 1654400 elements, which is an order of magnitude higher than the number of geomechanics elements currently employed. The flow domain is subject to no flux on all its boundaries whereas the geomechanics domain has a traction-free top surface and zero normal displacements on the remaining boundaries. The fluid compressibility is $1.45 \times 10^{-8} \mathrm{~Pa}^{-1}$, the fluid viscosity is $1 \mathrm{cp}$ and the initial reservoir porosity is taken to be 0.2 . The Biot parameter is taken to be 0.8 for the nearreservoir geomechanics elements and zero for the elements discretizing the overburden and underburden. The Young's modulus is taken to be $5.94 \times 10^{9} \mathrm{~Pa}$ and the Poisson's ratio is 0.2 . The initial reservoir hydrostatic pressure is taken to be $4650 \mathrm{psi}$, the reservoir permeability is taken to be $100 \mathrm{md}$ and the rock density is taken to be $2.65 \mathrm{~g} / \mathrm{cm}^{3}$. An injection well of BHP $7000 \mathrm{psi}$ is located at $y=2000 \mathrm{ft}, z=2000 \mathrm{ft}$ whereas a production well of BHP $2000 \mathrm{psi}$ is located at $y=6000 \mathrm{ft}, z=2000 \mathrm{ft}$. Figures 16, 17 and 18 are the results of the simulation on 128 processes with a time step of 1 day at the end of 200 days. The total run time was 15 minutes. The injection well generates an increase in pore pressure from the in situ hydrostatic pressure of $4650 \mathrm{psi}$ whereas the production well generates a decrease in pore pressure with radial flow patterns as shown in Figure 16. The concomitant geomechanical response is shown in Figure 17. As the $x$ axis points vertically downwards, positive $x$ displacements indicate movement in the direction of gravity whereas negative $x$ displacements indicate movement against the direction of gravity. The 

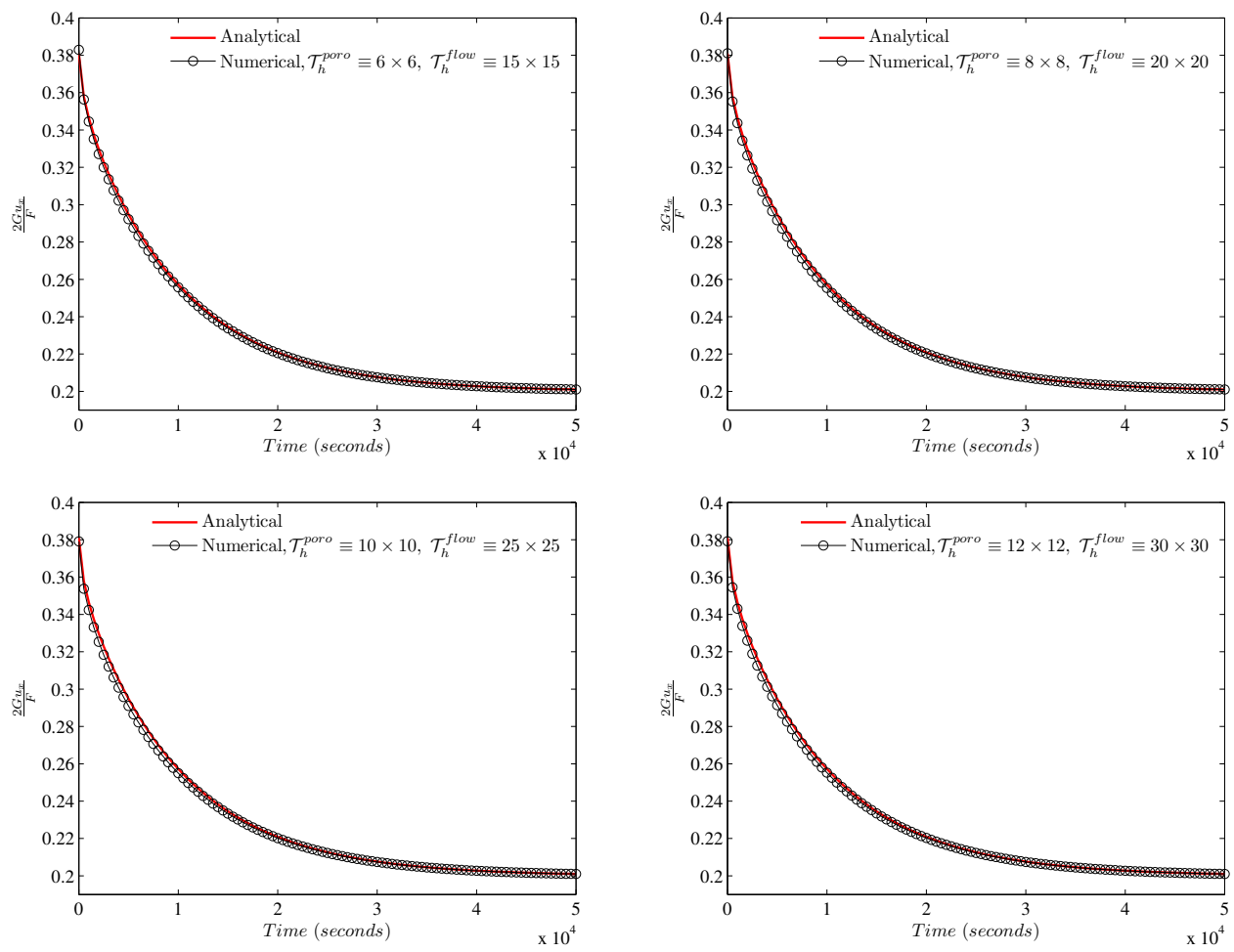

FIG. 14: Displacement response at the free end for the Mandel's problem with nonmatching grids. $\lim _{t \rightarrow 0^{+}}\left(\frac{2 G u_{x}(a, y, t)}{F}\right)=v_{u}=$ 0.3846 and $\lim _{t \rightarrow \infty}\left(\frac{2 G u_{x}(a, y, t)}{F}\right)=v=0.2$.

distribution of the vertical displacement on the free surface approximately $10000 \mathrm{ft}$ above the reservoir is shown in Figure 18. This distribution follows the radial flow patterns generated from the wells, while keeping in mind that the effect of fluid injection in the reservoir buried deep beneath the free surface is to induce an uplift whereas the effect of fluid withdrawal is to induce subsidence. As a result, portions of the geomechanical domain closer to the production well laterally experience larger movements in the direction of gravity resulting in larger positive values for $x$ displacement.

\section{SUMMARY}

Since flow and poromechanics are solved on different finite element grids, we design grid-to-grid projection operators using concepts in computational geometry and parallel computing. We first carve out a code framework in which flow is solved in parallel on a MPI subcommunicator and poromechanics is solved on the default MPI communicator. We then look at a pair of geometrically non-disjoint flow finite element and poromechanics finite element as two threedimensional objects intersecting one another. We then invoke singular value decompositions to obtain the equations of the surfaces of the flow and poromechanics elements. We then subject those equations to a predictor-corrector algorithm in order to perform surface-surface intersections. These intersections are used to obtain points on the periphery of the intersection polyhedron of that pair. We then employ a Delaunay triangulation routine that takes these points as input and provides the tetrahedralization of the intersection polyhedron. We add up the volumes of the tetrahedra to obtain the volume of the intersection polyhedron. This volume is then used to implement local grid-to-grid projection operators. The global grid-to-grid projection operators would be the assembly of the local operators. This entire code machinery is impemented as an addendum to the existing staggered solution algorithmic 


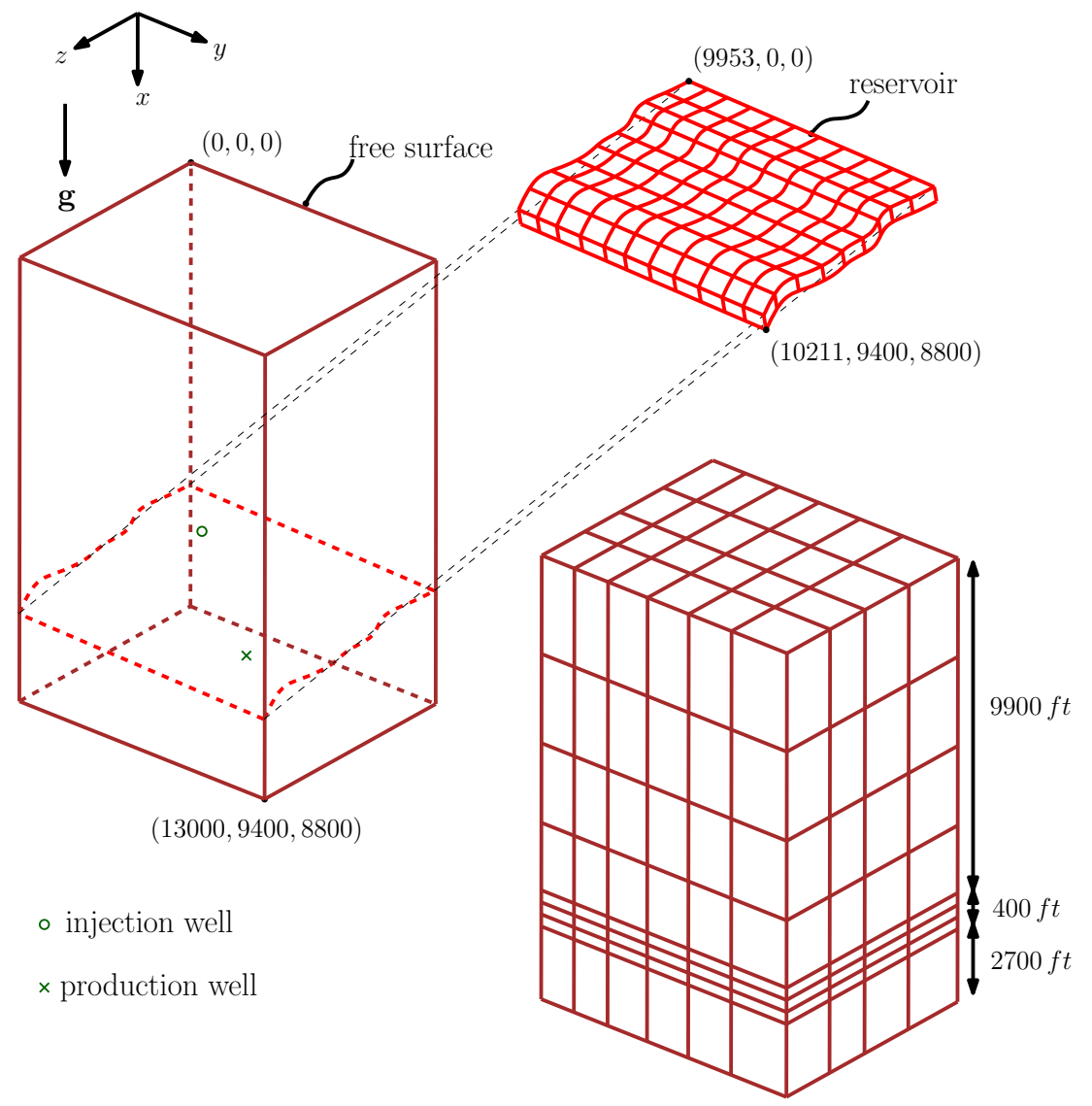

FIG. 15: Flow domain of $80 \mathrm{ft} \times 9400 \mathrm{ft} \times 8800 \mathrm{ft}$ surrounded by a geomechanics domain of $13000 \mathrm{ft} \times 9400 \mathrm{ft} \times 8800 \mathrm{ft}$. Injection well is located at $y=2000 \mathrm{ft}, z=2000 \mathrm{ft}$ and production well is located at $y=6000 \mathrm{ft}, z=6000 \mathrm{ft}$.

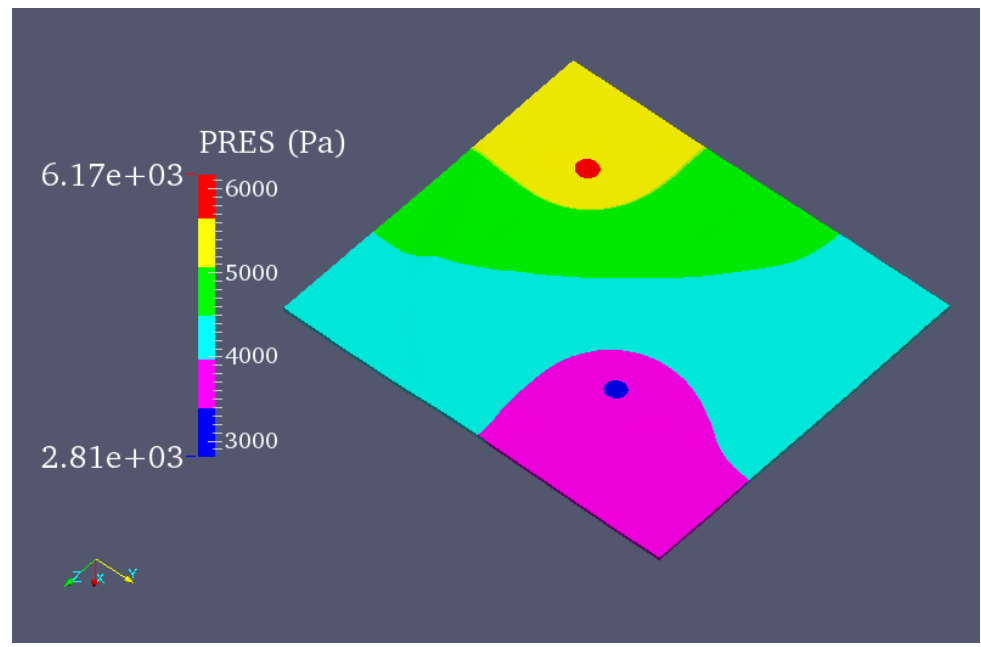

FIG. 16: Reservoir pore pressure distribution at the end of 200 days.

code framework that solves flow and poromechanics on the same finite element grid. We then establish the numerical 


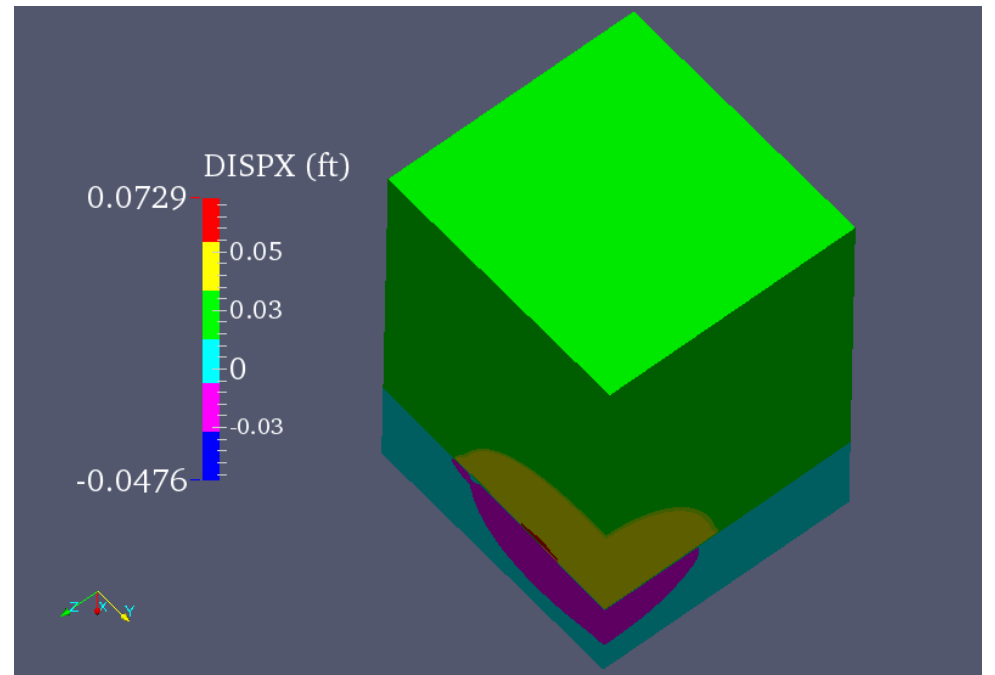

FIG. 17: Vertical displacement distribution in the geomechanical domain at the end of 200 days.

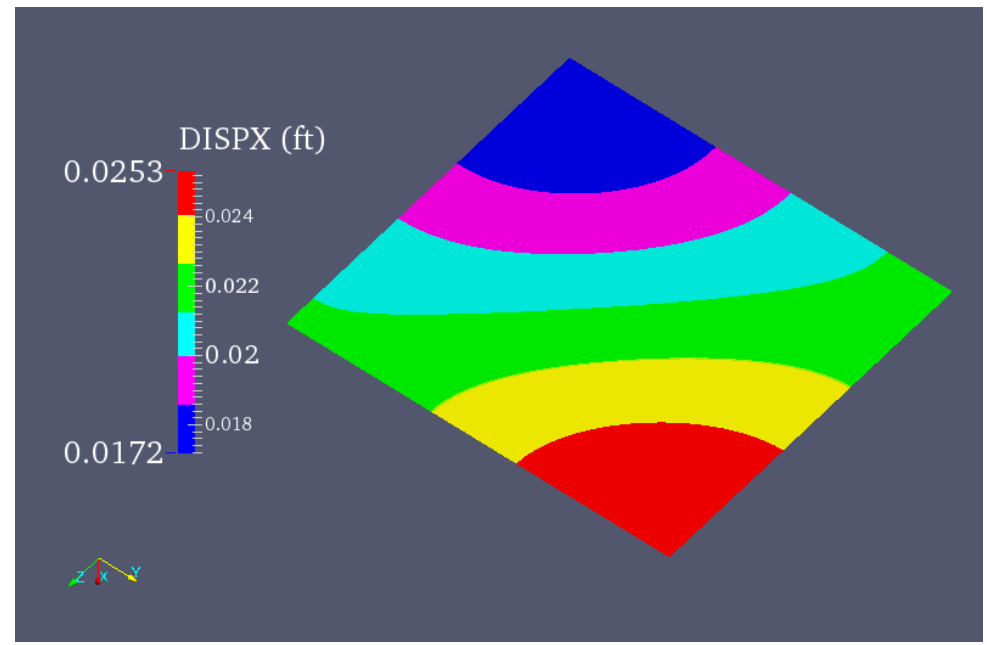

FIG. 18: Vertical displacement distribution at the free surface at the end of 200 days.

convergence of the solution algorithm for the benchmark problem, and demonstrate its capability in handling a largescale field problem. 


\section{References}

[1] Hiroo Kanamori and Emily E Brodsky. The physics of earthquakes. Reports on Progress in Physics, 67(8):1429, 2004.

[2] Christopher H Scholz, Yen Joe Tan, and Fabien Albino. The mechanism of tidal triggering of earthquakes at mid-ocean ridges. Nature communications, 10(1):1-7, 2019.

[3] Sebastian Hainzl, Toni Kraft, Joachim Wassermann, Heiner Igel, and E Schmedes. Evidence for rainfall-triggered earthquake activity. Geophysical Research Letters, 33(19), 2006.

[4] EK Montgomery-Brown, David R Shelly, and Paul A Hsieh. Snowmelt-triggered earthquake swarms at the margin of long valley caldera, california. Geophysical Research Letters, 46(7):3698-3705, 2019.

[5] ChiChing Liu, Alan T Linde, and I Selwyn Sacks. Slow earthquakes triggered by typhoons. Nature, 459(7248):833-836, 2009.

[6] Gillian R Foulger, Miles P Wilson, Jon G Gluyas, Bruce R Julian, and Richard J Davies. Global review of human-induced earthquakes. Earth-Science Reviews, 178:438-514, 2018.

[7] Youssef MA Hashash, Jeffrey J Hook, Birger Schmidt, I John, and Chiang Yao. Seismic design and analysis of underground structures. Tunnelling and underground space technology, 16(4):247-293, 2001.

[8] Jack Moehle. Seismic design of reinforced concrete buildings. McGraw-Hill Education, 2015.

[9] Mark D Zoback and Steven M Gorelick. Earthquake triggering and large-scale geologic storage of carbon dioxide. Proceedings of the National Academy of Sciences, 109(26):10164-10168, 2012.

[10] William L Ellsworth, Domenico Giardini, John Townend, Shemin Ge, and Toshihiko Shimamoto. Triggering of the pohang, korea, earthquake ( $\mathrm{m}$ w 5.5) by enhanced geothermal system stimulation. Seismological Research Letters, 90(5):1844-1858, 2019.

[11] Katie M Keranen and Matthew Weingarten. Induced seismicity. Annual Review of Earth and Planetary Sciences, 46:149$174,2018$.

[12] Saumik Dana and Mary F Wheeler. Augmented lagrangian for treatment of hanging nodes in hexahedral meshes. arXiv preprint arXiv:1809.04031, 2018

[13] Saumik Dana and Karthik Reddy Lyathakula. Uncertainty quantification in friction model for earthquakes using bayesian inference. arXiv preprint arXiv:2104.11156, 2021.

[14] S. Dana and M. F. Wheeler. Convergence analysis of fixed stress split iterative scheme for anisotropic poroelasticity with tensor biot parameter. Computational Geosciences, 22(5):1219-1230, 2018.

[15] S. Dana and M. F. Wheeler. Convergence analysis of two-grid fixed stress split iterative scheme for coupled flow and deformation in heterogeneous poroelastic media. Computer Methods in Applied Mechanics and Engineering, 341:788-806, 2018.

[16] Saumik Dana and Mary F Wheeler. Design of convergence criterion for fixed stress split iterative scheme for small strain anisotropic poroelastoplasticity coupled with single phase flow. arXiv preprint arXiv:1912.06476, 2019.

[17] Saumik Dana and Mary F Wheeler. An efficient algorithm for numerical homogenization of fluid filled porous solids: part-i. arXiv preprint arXiv:2002.03770, 2020.

[18] Saumik Dana and Mary F Wheeler. A machine learning accelerated $\mathrm{fe}^{2}$ homogenization algorithm for elastic solids. arXiv preprint arXiv:2003.11372, 2020.

[19] Saumik Dana, Benjamin Ganis, and Mary F. Wheeler. A multiscale fixed stress split iterative scheme for coupled flow and poromechanics in deep subsurface reservoirs. Journal of Computational Physics, 352:1-22, 2018.

[20] Saumik Dana, Shriram Srinivasan, Satish Karra, Nataliia Makedonska, Jeffrey D Hyman, Daniel O’Malley, Hari Viswanathan, and Gowri Srinivasan. Towards real-time forecasting of natural gas production by harnessing graph theory for stochastic discrete fracture networks. Journal of Petroleum Science and Engineering, 195:107791, 2020. 
[21] Saumik Dana, Joel Ita, and Mary F Wheeler. The correspondence between voigt and reuss bounds and the decoupling constraint in a two-grid staggered algorithm for consolidation in heterogeneous porous media. Multiscale Modeling \& Simulation, 18(1):221-239, 2020.

[22] Saumik Dana. A simple framework for arriving at bounds on effective moduli in heterogeneous anisotropic poroelastic solids. arXiv preprint arXiv:1912.10835, 2019.

[23] Saumik Dana. System of equations and staggered solution algorithm for immiscible two-phase flow coupled with linear poromechanics. arXiv preprint arXiv:1912.04703, 2019.

[24] S. Dana. Addressing challenges in modeling of coupled flow and poromechanics in deep subsurface reservoirs. PhD thesis, The University of Texas at Austin, 2018.

[25] O. Coussy. Poromechanics. Wiley, 2nd ed edition, 2004.

[26] M. A. Biot. General theory of three dimensional consolidation. Journal of Applied Physics, 12:155-164, 1941.

[27] A. Mikelić and M.F. Wheeler. Convergence of iterative coupling for coupled flow and geomechanics. Computational Geosciences, 17(3):455-461, 2013.

[28] N. Castelletto, J. A. White, and H. A. Tchelepi. Accuracy and convergence properties of the fixed-stress iterative solution of two-way coupled poromechanics. International Journal for Numerical and Analytical Methods in Geomechanics, 39(14):1593-1618, 2015.

[29] R. A. Adams. Sobolev spaces. Academic Press, 1975.

[30] F. Brezzi, J. Douglas, R. Durán, and M. Fortin. Mixed finite elements for second order elliptic problems in three variables. Numerische Mathematik, 51(2):237-250, 1987.

[31] R. Ingram, M.F. Wheeler, and I. Yotov. A multipoint flux mixed finite element method on hexahedra. SIAM Journal of Numerical Analysis, 48(4):1281-1312, 2010.

[32] T. J. R. Hughes. The Finite Element Method: Linear Static and Dynamic Finite Element Analysis. Dover Civil and Mechanical Engineering. Dover Publications, 2000.

[33] O. C. Zienkiewicz, R. L. Taylor, and J. Z. Zhu. The Finite Element Method: Its Basis and Fundamentals. ButterworthHeinemann, 7th edition, 2013.

[34] J. Geertsma. The effect of fluid pressure decline on volumetric changes of porous rocks. SPE, 210:331-340, 1957.

[35] R. J. S. Brown and J. Korringa. On the dependence of the elastic properties of a porous rock on the compressibility of the pore fluid. Geophysics, 40(4):608-616, 1975.

[36] C. L. Bajaj, C. M. Hoffmann, R. E. Lynch, and J. E. H. Hopcroft. Tracing surface intersections. Computer Aided Geometric Design, 5(4):285-307, 1988

[37] H. Si. Tetgen, a delaunay-based quality tetrahedral mesh generator. ACM Trans. on Mathematical Software, $41(2), 2015$.

[38] J. Mandel. Consolidation des sols (étude mathématique)*. Géotechnique, 3(7):287-299, 1953.

[39] C. W. Cryer. A comparison of the three-dimensional consolidation theories of biot and terzaghi. The Quarterly Journal of Mechanics and Applied Mathematics, 16(4):401-412, 1963.

[40] R. E. Gibson, K. Knight, and P. W. Taylor. A critical experiment to examine theories of three dimensional consolidation. In Eur. Conf. Soil Mech. Wiesbaden, volume 1, pages 69-76, 1963.

[41] A. Verruijt. Elastic storage of aquifers. In Flow through porous media (edited by R. J. M. DeWiest) New York: Academic Press, pages 331-376, 1965.

[42] Y. Abousleiman, A. H. D. Cheng, L. Cui, E. Detournay, and J. C. Roegiers. Mandel's problem revisited. Géotechnique, 46(2):187-195, 1996 
[43] A. Mikelić, B. Wang, and M. F. Wheeler. Numerical convergence study of iterative coupling for coupled flow and geomechanics. In 13th European conference on the Mathematics of Oil recovery, Biarritz, France, 2012.

[44] P.J. Phillips and M.F. Wheeler. A coupling of mixed and continuous galerkin finite element methods for poroelasticity ii: the discrete-in-time case. Computational Geosciences, 11(2):145-158, 2007. 